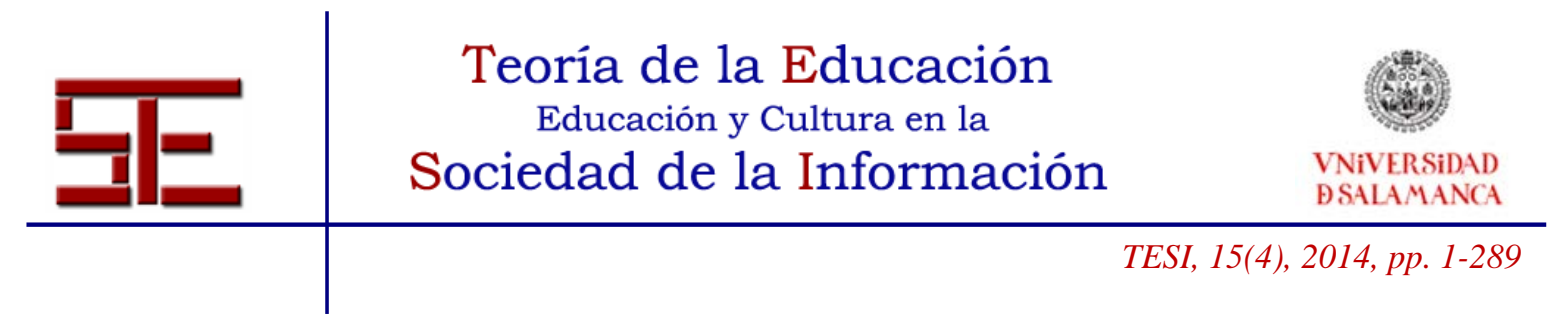

\title{
LA CONCRECIÓN DE LAS POLÍTICAS EDUCATIVAS DE INTEGRACIÓN DE LAS TIC EUROPEAS Y ESPAÑOLAS EN LA COMUNIDAD DE MADRID
}

\section{THE SPECIFICITY OF EUROPEAN AND SPANISH INTEGRATION EDUCATIONAL POLICIES ON ICT IN THE REGION OF MADRID}

\author{
Pablo SÁNCHEZ-ANTOLÍN \\ Universidad de Castilla La Mancha \\ pablo.sanchez@uclm.es
}

\author{
Joaquín PAREDES LABRA \\ Universidad Autónoma de Madrid \\ joaquin.paredes@uam.es
}

Resumen:

El artículo realiza un análisis de las políticas educativas sobre TIC en tres niveles, la Unión Europea, España y la región de Madrid.

Para ello se ha realizado un vaciado de los documentos marco y la normativa generados por instituciones relacionadas con las TIC y la educación en estos ámbitos. El análisis interniveles permite valorar la coherencia y ausencias más notables de las políticas puestas en marcha.

Se han identificado objetivos, acciones y valoración de su ejecución para cada política y las sinergias entre las mismas. Asimismo, se han valorado las implicaciones para el sistema educativo.

Entre las conclusiones de esta revisión se observa que las políticas que se sucedieron tuvieron altas miras y escaso sentido práctico, y sus resultados, concretados en la realidad de los centros, han sido modestos, más en la región de Madrid.

Palabras clave: Política educativa; Unión Europea; España; Madrid; TIC; Usos de ordenadores en las aulas.

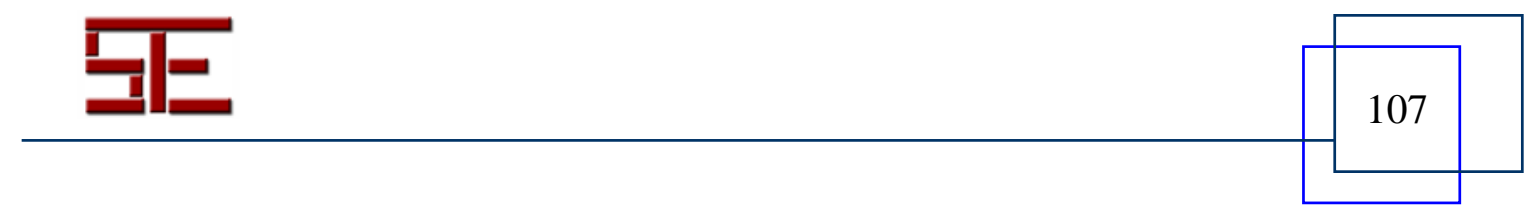


Abstract:

The paper aims to analyze the educational policies on ICT in three levels: the European Union, Spain, and the region of Madrid.

Bearing in mind this aim, we study the framework documents and the regulation provided by institutions working on ICT and education within these areas. The interlevel analysis allows us to assess the coherence and notable absences of the policies implemented.

We have identified objectives, actions and assessment during the process of implementation for each policy and the synergies generated among them. Moreover, We analyze the implications for the educational system.

We underline some conclusions taking as a reference this evaluation. The study implemented display that the policies were very ambitious theoretically and low practical sense, and its results, specified in the reality of the centers, have been modest, even more in the region of Madrid.

Key words: Educational policy; European Union; Spain; Madrid; ICT; Uses of computers in classrooms.

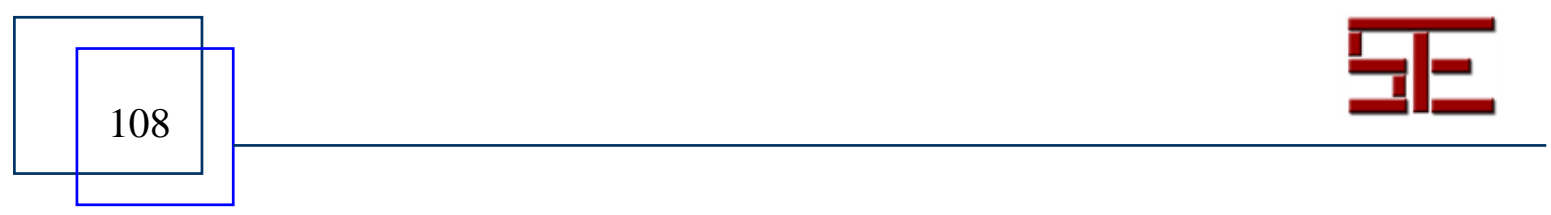




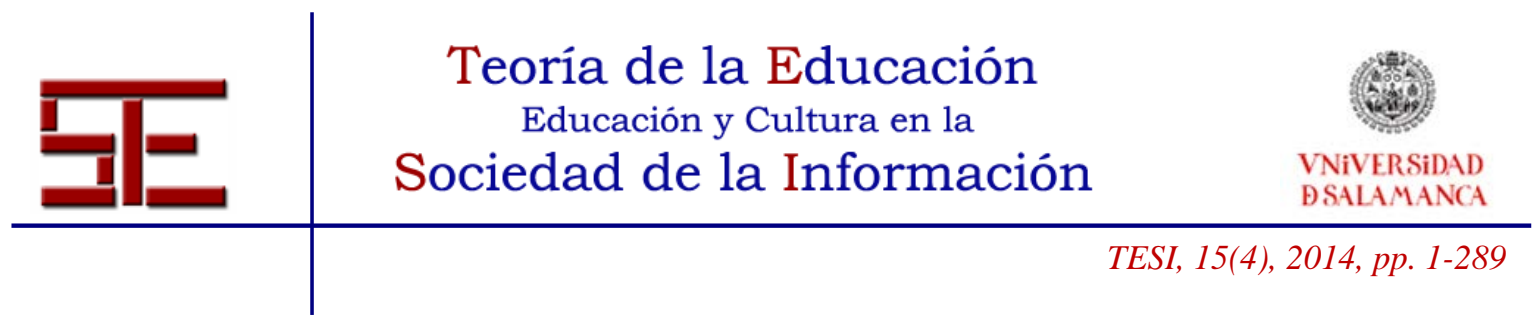

\section{INTRODUCCIÓN}

Desde hace más de tres décadas los responsables políticos han considerado que las tecnologías de la información y comunicación (TIC) poseen un gran potencial para transformar y modernizar la educación (OECD, 2010) y que, por tanto, era necesaria su introducción en los sistemas educativos. En el contexto de la Unión Europea, se han sucedido los planes de acción y programas que inciden en su integración y que han tenido su reflejo en las políticas nacionales y regionales.

Estas políticas han soñado con la idea de que las TIC podrían ser la solución a los problemas fundamentales de la educación (individualizarla para mejorar las oportunidades de aprendizaje, mejorar el rendimiento del alumnado, elevar la calidad de la educación a menor coste...) y se han presentado como la "gran esperanza blanca de la educación” (Sancho, Ornellas, Sánchez, Alonso, y Bosco, 2008, 11), como el talismán que puede proporcionar resultados extraordinarios (Somekh, 2000, 20-21), o como la panacea que puede revolucionar la educación (Burbules y Callister, 2001, 24). Además, se pensaba, y se piensa, que éstas pueden convertirse en un componente clave que hay que aprovechar para lograr un mayor crecimiento económico y social (Cela, 2005, 189149).

Con esta idea, se comenzaron a aplicar políticas de introducción de las TIC en los sistemas educativos. Así, desde los años 80 en Inglaterra y el resto de Europa (como luego se dirá) y desde 1993 en Estados Unidos, primero con el Plan Gore y después, en 1996, con el anuncio de la revolución educativa digital de Bill Clinton, se vienen realizando planes tecnológicos con el objetivo de explotar sus potencialidades que, en la mayoría de los casos, incluyen objetivos para dotar con ordenadores al alumnado, conectar las escuelas a Internet y entre sí, la incorporación de software educativo al currículum y la formación del profesorado (Piscitelli, 2010, 5).

En los últimos años y desde que en el año 2002 el Estado de Maine pusiese en marcha la primera iniciativa a gran escala de un ordenador por cada alumno (modelo 1:1) (Instituto de Tecnologías Educativas, 2011), se ha extendido este tipo de políticas, tanto en Europa como en Iberoamérica, sobre todo, debido a la reducción de costes de los equipos informáticos, su reducido peso y la disponibilidad de conectividad inalámbrica (Valiente González, 2011, 116). Todo ello a pesar de que no existen suficientes evidencias que expliquen qué fenómenos y factores, no sólo asociados a la introducción masiva de las TIC en los centros y aulas, favorecen los procesos de innovación y mejora educativa que se pueden producir trabajando con ordenadores (Area Moreira, 2010). Se ha optado por "promover, incentivar y potenciar tanto como sea posible el equipamiento tecnológico, así como pavimentar cuantos kilómetros se pueda de la supercarretera de la información que Gore y Clinton anunciaban con tanto entusiasmo hace más de quince

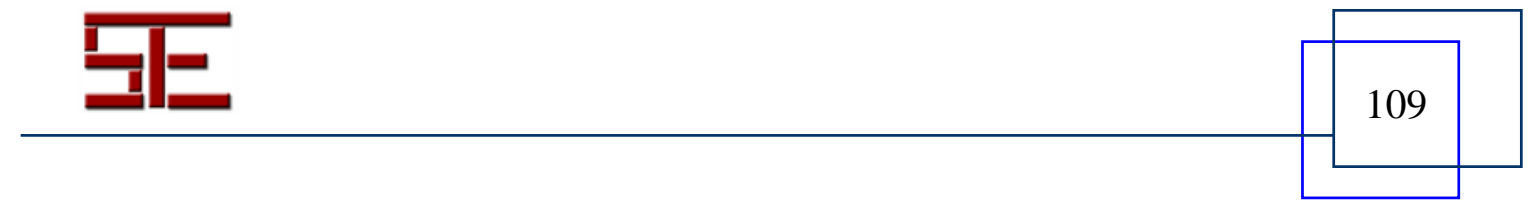




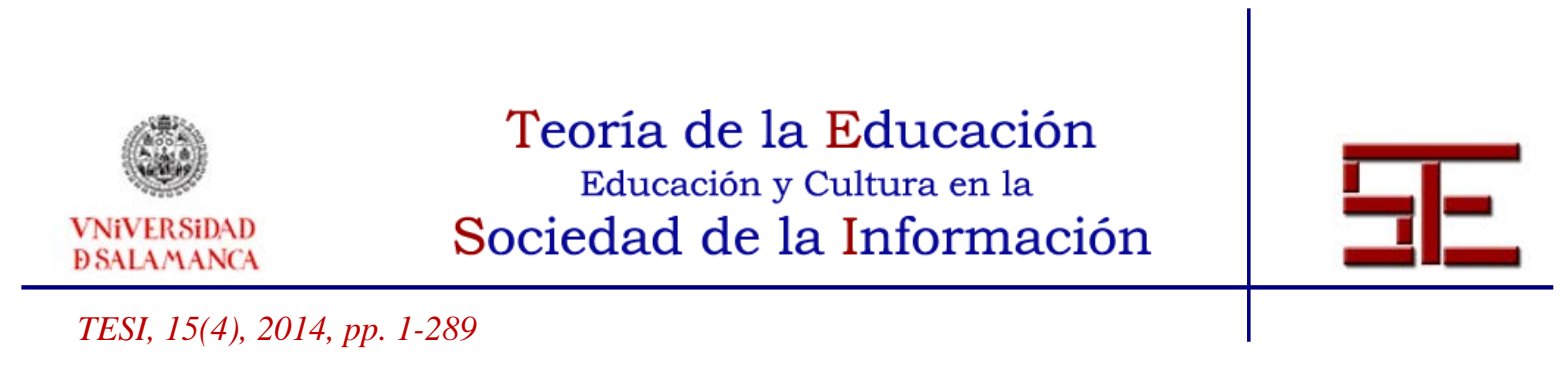

años” (Cobo Romaní, 2010, 132).

\section{LAS POLÍTICAS EUROPEAS SOBRE SOCIEDAD DE LA INFORMACIÓN}

Europa, para no quedarse rezagada ante los nuevos retos de la sociedad de la información, en las últimas tres décadas, ha implementado políticas tanto a nivel europeo, como nacional y regional, destinadas a la integración de las TIC en los sistemas educativos. Estas políticas, conscientes de que necesitan buenos sistemas educativos, con modernas herramientas tecnológicas, entre otros aspectos, han incidido en la alfabetización digital de los ciudadanos y en facilitar posibilidades de aprendizaje permanente (Soto Carballo, 2007) que propicien alcanzar unos niveles educativos básicos y polivalentes que permitan el progreso económico, la cohesión social y el desarrollo democrático (Area Moreira, 2012a).

Las primeras referencias a la importancia que tiene la educación en la sociedad de la información las encontramos en el informe Europa y la sociedad mundial de la información (Bangemann, 1994) y un año después en el Libro Blanco sobre la Educación y la formación: enseñar y aprender, hacia la sociedad cognitiva. En el primero, se hace alusión a la necesidad de formar y alfabetizar informáticamente al profesorado; y en el segundo, se dice que lo "ideal sería llegar a dotar a cada clase de los equipos necesarios para que los jóvenes accedan a la informática. Esto implica, concretamente, que Europa se dote de nuevos instrumentos pedagógicos de calidad, adaptados a sus tradiciones educativas y culturales" (Comisión de las Comunidades Europeas, 1995, 32). También se reconoce el papel central del profesorado y la necesidad de modificar sus prácticas pedagógicas, de forma que los individuos sean capaces de desarrollar un sentido crítico que les permita protegerse de la manipulación e ideologías dominantes de los "profesores salvajes” (Comisión de las Comunidades Europeas, 1995, 10) que son los medios de comunicación y las redes informáticas.

\subsection{Avanzando hacia la conectividad y la capacitación digital}

Una de las propuestas más importantes en las políticas europeas de accesibilidad electrónica fue eEurope, iniciativa que se puso en marcha en 1999 y que fue adoptada por el Consejo Europeo de Lisboa de Marzo de 2000, en la que se fijó como objetivo estratégico para Europa "convertirse en la economía basada en el conocimiento más competitiva y dinámica del mundo con más y mejores empleos y con mayor cohesión social” (Consejo de Europa, 2000, 2) antes del año 2010.

Los objetivos generales que se planteaban trataban de dar respuesta a los problemas que

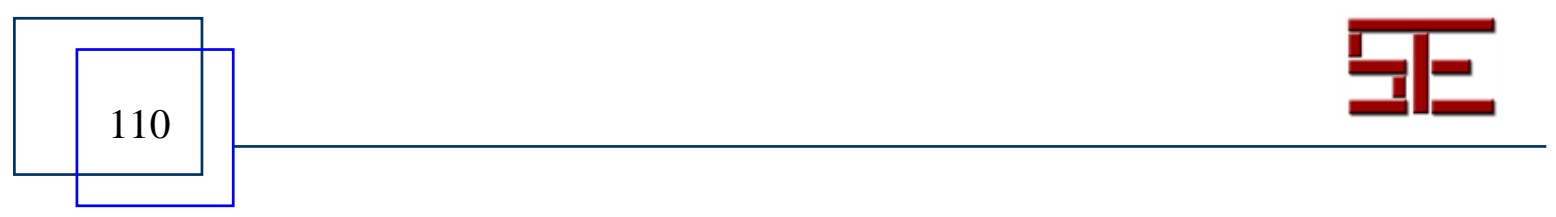




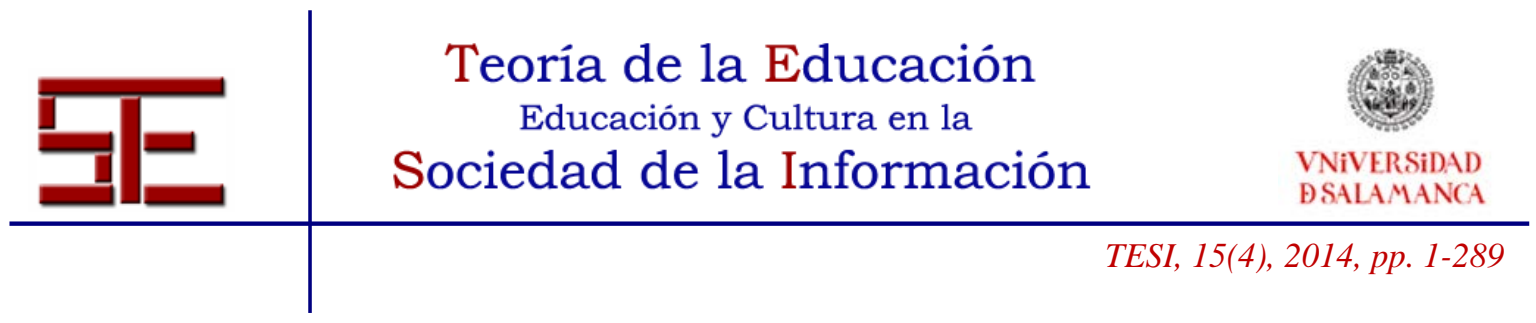

estaban impidiendo un mayor desarrollo de la sociedad de la información en Europa: accesos insuficientes a Internet, una población con insuficiente formación digital, una administración que no estaba realizando los esfuerzos suficientes para facilitar el desarrollo de nuevas aplicaciones y servicios y la falta de una cultura dinámica y emprendedora orientada a la prestación de servicios.

De las prioridades inicialmente planteadas por la acción eEurope, tres afectaban directamente a la educación de jóvenes y adultos: la adquisición de competencias para desempeñar un papel activo en la sociedad de la información, la mejora de Internet para los investigadores y estudiantes europeos; y la accesibilidad de las personas con discapacidad a la cultura electrónica. La primera de ellas destaca la educación como elemento fundamental para el progreso económico y social y la igualdad de oportunidades. El acceso de la juventud a la era digital necesita de una formación que permita el dominio de internet y los recursos multimedia, la utilización de las tecnologías para aprender y la adquisición de competencias de trabajo en equipo, creatividad, comunicación intercultural, capacidad de adaptación y aptitud para resolver problemas. Entre los objetivos que se fijan para el cumplimiento de esta prioridad se encuentran: la conexión de las escuelas y del alumnado a Internet en las aulas; que el alumnado finalice su formación básica capacitado digitalmente y la capacitación del profesorado, ya que el éxito de esta iniciativa depende en gran medida del grado de participación de éste y de la dirección de las escuelas.

\subsection{De la inclusión digital a la mejora de la productividad y el fomento de la competitividad}

Para lograr los objetivos planteados por la iniciativa eEurope, en el año 2000 se puso en marcha el plan de acción eEurope 2002 (Comisión de las Comunidades Europeas, 2000), que agrupaba las prioridades enunciadas por eEurope en tres objetivos y se completaba con otras iniciativas que le apoyaban, pero no estaban integrados en el mismo: eLearning (en el campo de la formación continua y, años más tarde, eTwinning, que nació dentro de este programa con el objetivo de animar a las escuelas a colaborar utilizando las TIC), goDigital (en el campo de la integración de las pymes en la sociedad de la información) y eContent (en el campo del desarrollo y comercialización de contenidos digitales).

Este plan de acción pretendía aprovechar el potencial de las tecnologías de la información para evitar la exclusión digital de las personas que no se benefician plenamente de la sociedad de la información por diversas razones (pobreza, falta de sensibilización y formación al respecto o discapacidad), en una sociedad en la que cada vez se van a ofrecer más servicios públicos e información de importancia en línea. Los retos que se planteaban eran lograr una mayor accesibilidad a las tecnologías y

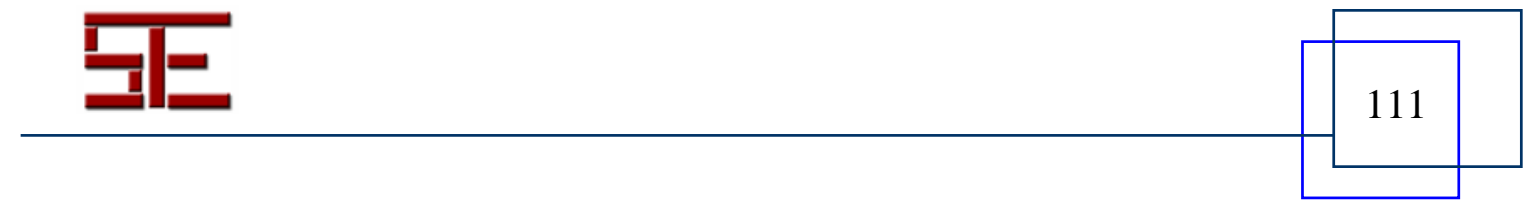




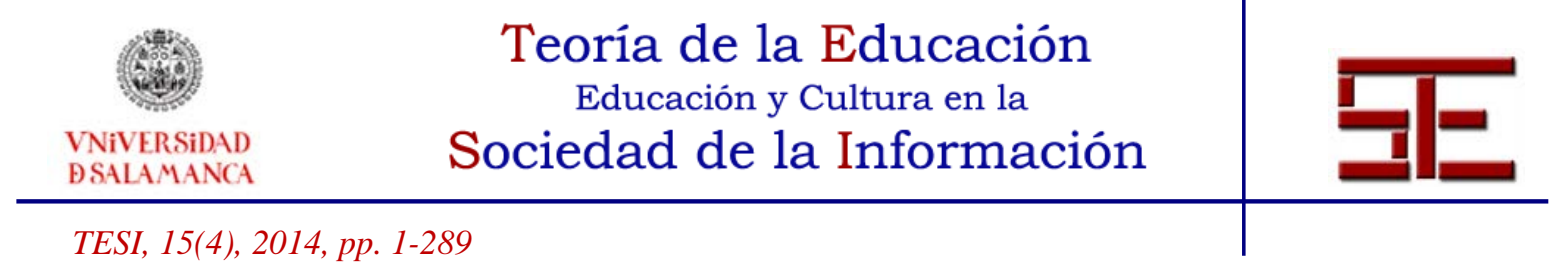

garantizar su compatibilidad con las tecnologías de ayuda.

Tomando como punto de partida la evaluación de eEurope 2002 y las recomendaciones del Consejo de Europa de 2002, se formuló el plan de acción eEurope 2005, que tuvo como objetivo principal "fomentar unos servicios, aplicaciones y contenidos seguros basados en una infraestructura de banda ancha ampliamente disponible" (Comisión de las Comunidades Europeas, 2002, 9) y entre los que se incluía como tema transversal la inclusión digital y participación ciudadana, que se tradujo en garantizar el acceso a los servicios TIC y la disponibilidad de estas tecnologías a un precio asequible para las personas con discapacidad y una alfabetización digital que permita dotar a todos los ciudadanos de las habilidades necesarias para hacer un uso completo y eficaz de las mismas (Comisión de las Comunidades Europeas, 2004a).

Los resultados moderados de estas iniciativas se vieron primeramente en el informe de evaluación de Wim Kok (2004) y en los resultados de las evaluaciones intermedias (Comisión de las Comunidades Europeas, 2004b) que se iban realizando del plan de acción eEurope 2005, que con posterioridad quedaron reflejados en el informe final (Comisión de las Comunidades Europeas, 2009). Los objetivos principales de eEurope 2002 y 2005, centrados en promover las redes y las conexiones a Internet para alcanzar unos altos niveles de desarrollo de la sociedad de la información, se revelaron insuficientes.

Esto propició el lanzamiento de i2010, iniciativa que aborda de manera integrada la sociedad de la información y las políticas audiovisuales en la Unión Europea. En este relanzamiento de la estrategia de Lisboa, en sus directrices se incluyeron objetivos que pretendían mejorar e impulsar la educación de los ciudadanos europeos. Entre los referidos a la educación se incluían la adaptación de la educación y formación a las nuevas necesidades en materia de competencias, el reforzamiento de equipamientos y conectividad para los centros educativos, la formación y asesoramiento para docentes formadores en el uso de las TIC y el desarrollo de contenidos digitales de calidad (Segura, Candioti, y Medina, 2007, 20).

La iniciativa i2010 se estructuró en torno a tres ejes: un espacio único Europeo de la Información que ofrezca comunicaciones de banda ancha asequibles y seguras, contenidos ricos y diversificados y servicios digitales; rendimiento de nivel mundial en la investigación y la innovación en el ámbito de las TIC, acercando así a Europa a sus principales competidores; y una sociedad de la información que sea incluyente, ofrezca servicios públicos de gran calidad y promueva la calidad de vida (Comisión de las Comunidades Europeas, 2005, 4). El último de ellos, el de carácter más social, trata de conseguir tres objetivos: "que las TIC beneficien a todos los ciudadanos, que los servicios públicos sean mejores, más rentables y más accesibles y que mejoren la calidad de vida" (Comisión de las Comunidades Europeas, 2005, 10). Por tanto, la einclusión (una sociedad de la información para todos) se vuelve a convertir en uno de

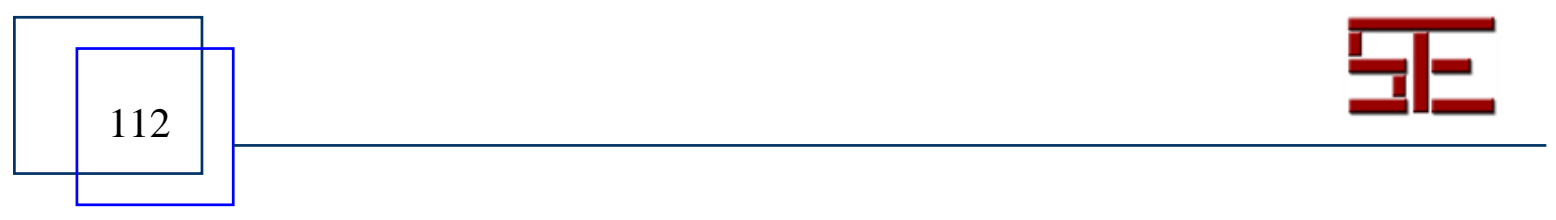




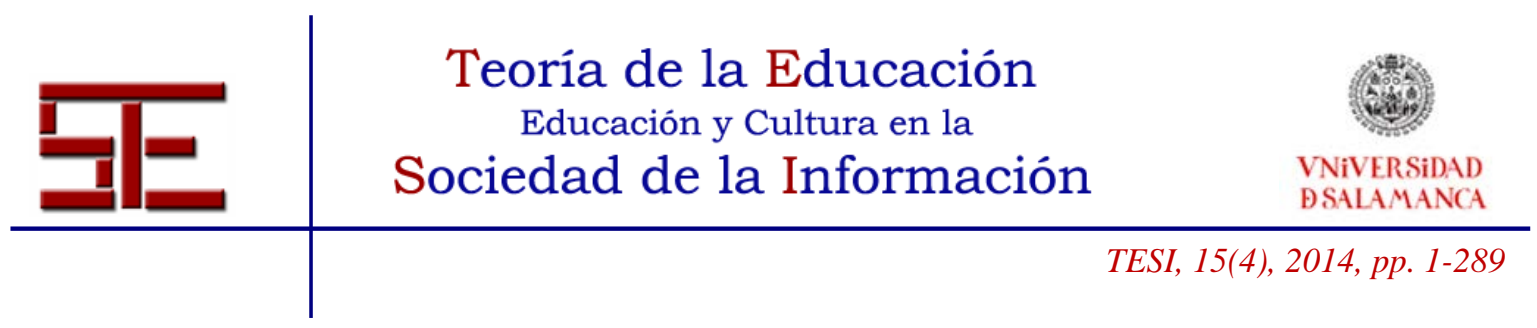

los objetivos prioritarios de las políticas europeas, tratando aspectos como el envejecimiento de la población, la accesibilidad, las brechas digitales, la administración electrónica, y la alfabetización y cultura digitales (Comisión de las Comunidades Europeas, 2006).

Un hecho decisivo en el reconocimiento de la importancia de la e-inclusión fue la Conferencia Ministerial de Riga de 2006 (TIC para una sociedad inclusiva) (Comisión Europea, 2006) en la que se establecieron objetivos concretos respecto a la disponibilidad de Internet, la alfabetización digital y la accesibilidad. Dichos objetivos debían de cumplirse antes de que finalizase 2010, pero en la Comunicación de la Comisión de 2007 Participar en la sociedad de la información (Comisión de las Comunidades Europeas, 2007) se advierte de la existencia de grandes disparidades dentro de Europa que harán muy difícil el cumplimiento de estos objetivos, incluso se advierte de que las diferencias sociales en el uso de las TIC, en algunos casos, están agravándose.

Entre los retos que plantea, aunque se reconoce que en algunas regiones se han disminuido las diferencias, están la toma de medidas que permitan aumentar la participación activa en la sociedad de la información de los ciudadanos europeos y den respuesta a la poca oferta de tecnologías accesibles, el aumento de la brecha digital, las diferencias en la disponibilidad de ordenadores y conexión de banda ancha en las escuelas, y en las competencias digitales del personal docente, entre otros.

En 2010, la Comisión Europea, reconoció que un 30\% de los europeos nunca ha utilizado Internet y que la penetración de la fibra óptica es de tan sólo el 1\%, datos que revelan el escaso éxito de las iniciativas eEurope e incluso i2010 (Echeverría y Unceta, 2012). A estos datos hay que añadir el reconocimiento de la insuficiente alfabetización digital de la ciudadanía:

Europa padece una creciente penuria de cualificación profesional en las TIC y un déficit en la alfabetización digital. Estas carencias están excluyendo a muchos ciudadanos de la sociedad y la economía digitales y limitando el gran efecto multiplicador que puede tener la adopción de las TIC sobre el aumento de la productividad. Se precisa una reacción coordinada, centrada en los Estados miembros y en otras partes interesadas (Comisión Europea, 2010a, 7).

La crisis económica ha transformado a Europa y los objetivos de las iniciativas anteriores destinados a mejorar las infraestructuras de las escuelas, la formación del profesorado, la competencia digital del alumnado para evitar su exclusión digital y fomentar su participación cívica, se han abandonado en favor de otras prioridades que buscan disminuir el desempleo juvenil, formando a jóvenes altamente cualificados, con capacidad de movilidad y con unos aprendizajes que les permitan adquirir las competencias básicas para adaptarse e incorporarse rápidamente a las necesidades de los mercados.

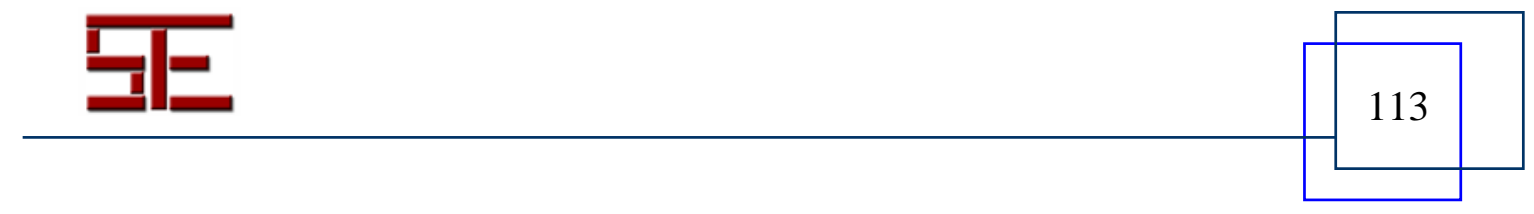




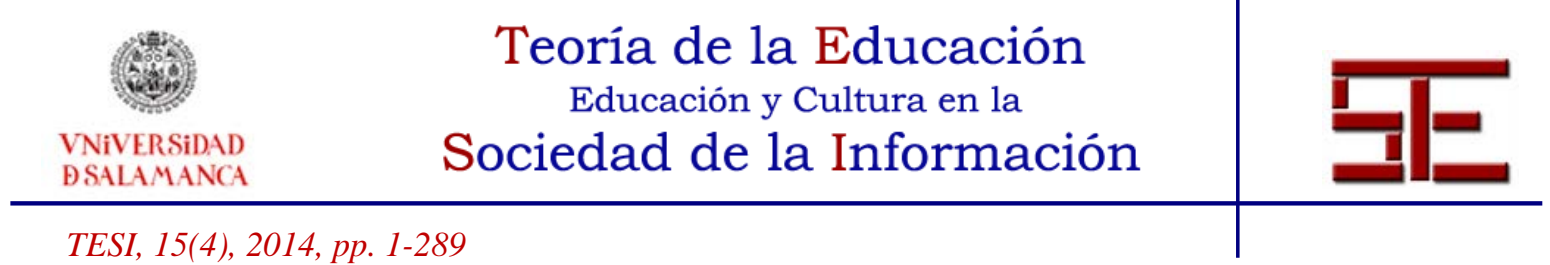

\subsection{Europe 2020 y Replantear la Educación}

Europe 2020 es la estrategia que fue aprobada por la Comisión Europea en 2010 y que sustituye a la de Lisboa e incluye siete iniciativas: Unión por la innovación; Juventud en movimiento; Una agenda digital para Europa; Una Europa que utilice eficazmente los recursos; Una política industrial para la era de la mundialización; Una agenda para nuevas cualificaciones y empleos; y Una plataforma europea contra la pobreza (Comisión Europea, 2010b, 4).

El objetivo de la Agenda Digital para Europa es “obtener los beneficios económicos y sociales sostenibles que pueden derivar de un mercado único digital basado en una internet rápida y ultrarrápida y en unas aplicaciones interoperables” (Comisión Europea, 2010a, 3). Propone medidas que favorezcan el desarrollo, sobre todo de Internet, como soporte de la actividad económica y social: para hacer negocios, trabajar, jugar, comunicarse y expresarse en libertad. En conjunto, trata de fomentar la innovación, el desarrollo económico y la mejora de la vida cotidiana de empresas y ciudadanos.

Las medidas educativas que se proponen desde la Comisión Europea tienen por objetivo disminuir la brecha y exclusión digital y modernizar la educación y la formación, pero no tienen en cuenta la educación para la e-participación, entendida como toma de decisiones, sino que sólo se piensa en el desarrollo de las tecnologías y servicios que permitan el e-gobierno, entendido como realización de gestiones administrativas (Echeverría y Unceta, 2012).

Rethinking Education (Replantear la Educación), se lanzó a finales de 2012 por la Comisión Europea y tiene como objetivo propiciar el desarrollo de las competencias y capacidades que son necesarias para el mercado de trabajo. Entre las medidas que hacen referencia a las TIC se encuentran el aprovechamiento de los recursos educativos abiertos (REA), que deben incluir mecanismos que permitan la evaluación y las competencias adquiridas con estos materiales; así como la modernización de las infraestructuras TIC en las escuelas (Gobierno de España, 2013b).

\subsection{Erasmus+ el último programa de Unión Europea}

La mayoría de las medidas educativas que la Unión Europea planteaba hasta este momento se concretaban en la iniciativa Juventud en movimiento que, en 2014, ha sido incorporada al programa Erasmus+ (Comisión Europea, 2014). Este nuevo programa, se enmarca en las estrategias Europe 2020, Educación y Formación 2020 y Rethinking Education y abarca todos los niveles educativos. En general, persigue la promoción de la movilidad de estudiantes, incrementar la calidad general de todos los niveles educativos y de formación y mejorar la empleabilidad de los jóvenes.

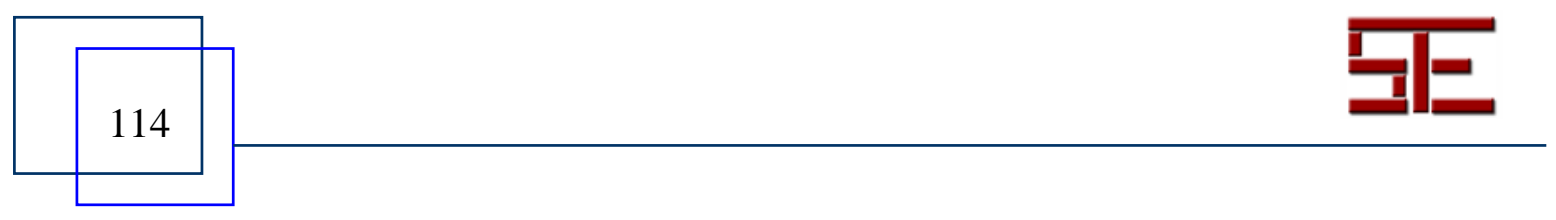




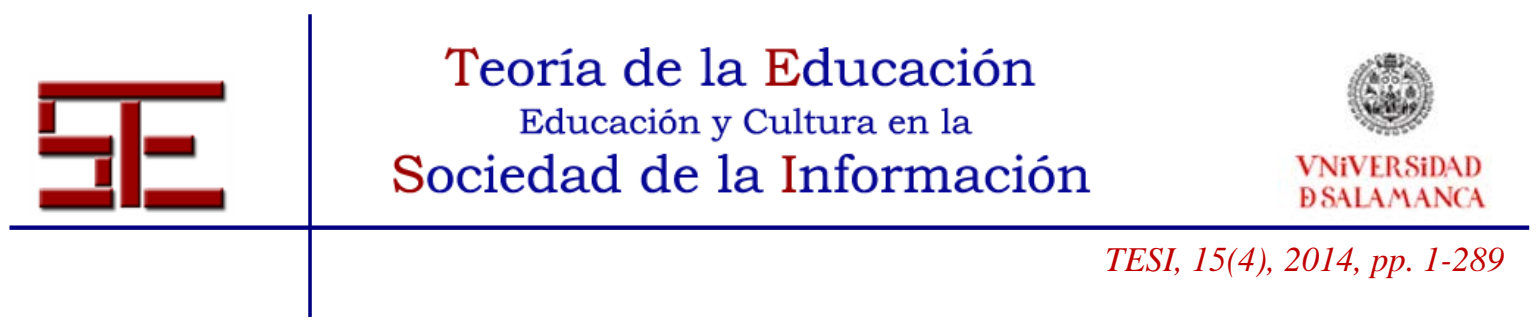

Respecto a la educación y formación, el programa Erasmus+ perseguirá, entre otros objetivos el "mejorar el nivel de las aptitudes y competencias clave, atendiendo en particular a su pertinencia para el mercado de trabajo y a su contribución para la cohesión social, en particular a través de un incremento de las oportunidades de movilidad por motivos de aprendizaje así como a través de una cooperación reforzada entre el mundo de la educación y la formación y el mundo del trabajo" (Diario Oficial de la Unión Europea, 2013, L 347/57).

Apoyada en las estrategias Replantear la Educación, la Agenda Digital Europea y European Higher Education in the World, a finales de 2013 y enmarcado dentro del programa Erasmus+, se presentó la iniciativa Apertura de la Educación (Opening up education) que entre sus objetivos vuelve a promover la innovación en la enseñanza a través del uso de las TIC, la formación del profesorado, la mejora de las infraestructuras TIC en los centros docentes, el uso compartido de recursos educativos y la cooperación entre organizaciones (Instituto Nacional de Tecnologías Educativas y de Formación del Profesorado - INTEF, 2013a).

Esta última iniciativa no aporta ideas sustantivas en el ámbito educativo ya que mantiene los objetivos de las iniciativas y estrategias anteriores. Su propósito sigue siendo aumentar las habilidades digitales del alumnado de forma que, una vez finalizado el periodo educativo, se mejore su futura empleabilidad y se adapten mejor a las necesidades del mercado. También lo son la mejora de la competencia digital del profesorado y de las infraestructuras de los centros.

\section{LAS POLÍTICAS TIC ESPAÑOLAS}

De estos objetivos e iniciativas propuestos desde la Unión Europea (UE) a través de los diferentes programas, estrategias y planes de acción, se derivan fondos para todos los países integrados en la UE. Para España se han centrado en la movilidad de los estudiantes, la creación de entornos de aprendizaje abierto y a distancia, la creación de redes educativas, el aprendizaje de lenguas, el aprovechamiento pedagógico de las TIC (Valle López, 2004), la dotación de tecnologías a los centros educativos, la alfabetización digital de la ciudadanía, la innovación educativa, la difusión de buenas prácticas, la creación de contenidos digitales y la formación del profesorado. Medidas que han tenido y tienen una traducción directa en las políticas españolas, no sólo en los planes y programas de impulso a la sociedad de la información, en los que se incluyen actuaciones que afectan al sistema educativo, sino también en las propias leyes educativas (LOGSE, LOCE, LOE, LOMCE), en las que se insiste en que la orientaciones políticas de nuestros sistema educativo tengan una estrecha relación con las directrices propuestas desde la UE (Ferreiro Alonso, 2011; Madrid Izquierdo, 2007).

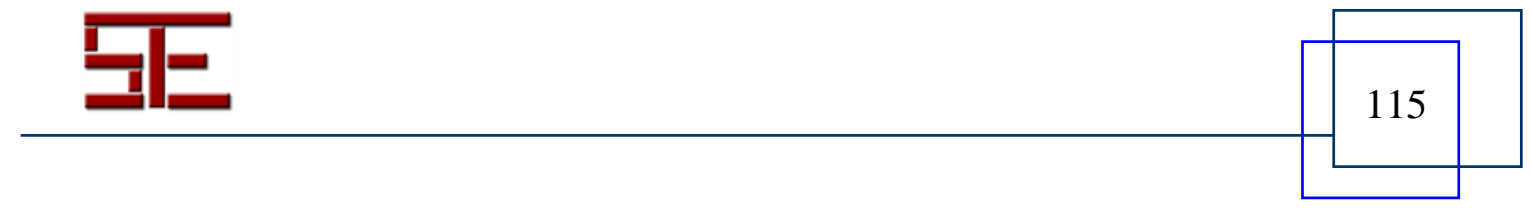




\section{\begin{tabular}{c} 
Teoria de la Educación \\
Educación y Cultura en la \\
$\begin{array}{c}\text { VNiVERSIDAD } \\
\text { BSALAMANCA }\end{array}$ \\
\hline TESI, 15(4), 2014, pp. 1-289
\end{tabular}}

\subsection{Planes nacionales para la introducción de las TIC en los centros educativos}

Al igual que en la mayoría de los países europeos, en España las políticas de integración de las TIC comenzaron a mediados de los años 80 con los programas Atenea, que tenía como objetivo incorporar equipos y programas informáticos a los centros educativos en un contexto de innovación educativa (Fernández Prieto, 2001) y Mercurio, que proponía la incorporación de los medios audiovisuales en las aulas, en especial el vídeo (del Blanco Diez, 1989), y fueron puestos en marcha por Ministerio de Educación y Ciencia e integrados en el año 1987 en el PNTIC (Programa de Nuevas Tecnologías de la Información y de la Comunicación).

Estos programas se fundieron en los años 90 y se caracterizaron por ser una propuesta dotacional, un observatorio de materiales y tendencias, un espacio para la formación permanente (la inicial estaba en mano de las universidades), un repositorio de materiales generados por los propios docentes y un espacio para diversas iniciativas, la formación ocupacional a distancia, los hermanamientos... El proceso de descentralización del país entregó las unidades regionales y una forma de trabajar a las Comunidades Autónomas. Todas las regiones, conviene indicarlo, han tenido históricamente una voluntad de cooperación y búsqueda conjunta de soluciones a problemas comunes a través de los servicios autónomos que fueron alcanzando sucesivamente.

En 2004, a partir de las recomendaciones de la Comisión Soto, y como parte del programa España.es, impulsado por el Gobierno de España, se puso en marcha Internet en la escuela que se proponía mejorar las infraestructuras TIC de los centros educativos, la formación de los docentes, la creación de portales educativos con contenidos digitales, comunidades virtuales, etc. Esta iniciativa respondía a los objetivos que se marcaban desde la iniciativa europea eEurope 2005.

Un año después, se puso en marcha el Plan Avanz@, también impulsado por el Gobierno de España, que respondía a los objetivos de la iniciativa europea i2010 y en la que se inició el programa Internet en el aula. Este nuevo programa trataba de dar continuidad a Internet en la escuela y desarrolló medidas dirigidas a favorecer, de una parte, la utilización de las TIC como herramienta de apoyo en el proceso de enseñanzaaprendizaje y, de otra, al fomento de la utilización de las redes telemáticas como vehículo de comunicación, entendimiento y cooperación entre los participantes en el proceso educativo (Segura et al., 2007, 24). Estas medidas contemplaban la dotación de equipamiento TIC, incluidos equipos adaptados al alumnado con necesidades educativas especiales, la conectividad de los centros, la creación de centros públicos de acceso a Internet en los centros educativos, servicios de formación y orientación a las familias en TIC, creación y recopilación de contenidos para la comunidad educativa, etc. (Gobierno de España, 2005, 12-16).

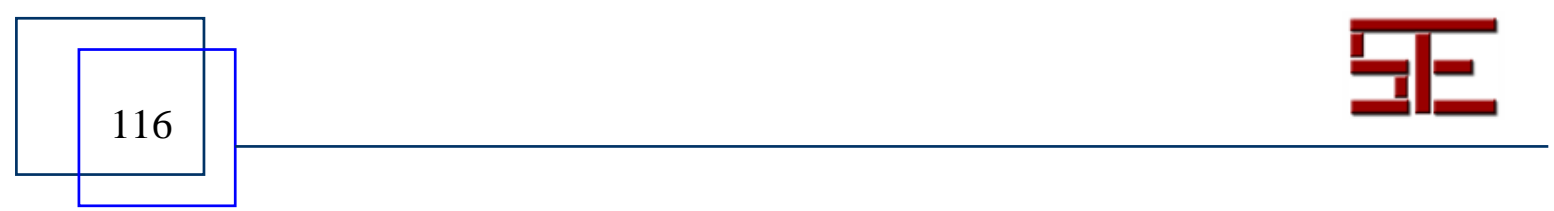




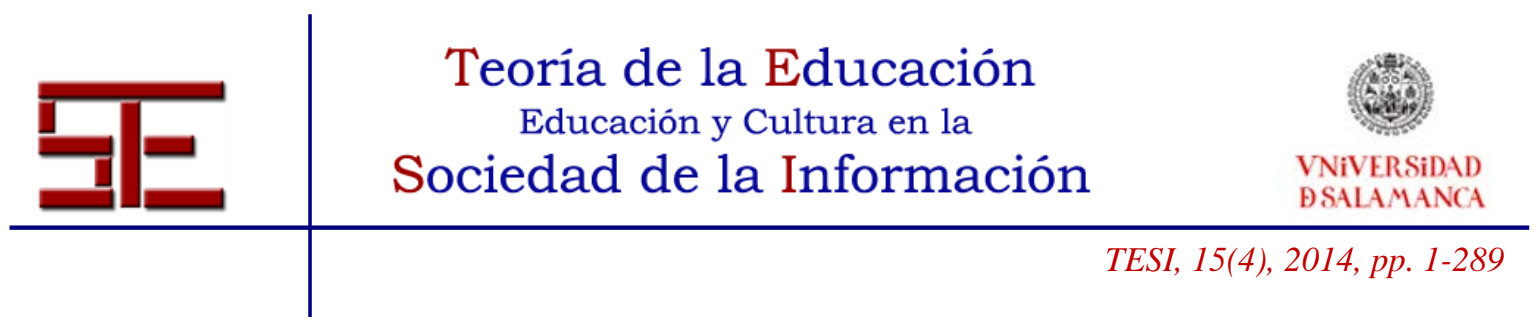

Hasta el 2009 las políticas educativas de integración de las TIC habían dotado con ordenadores los centros educativos para tareas administrativas, las aulas con pizarras digitales y los PC para el profesorado, aulas y laboratorios de informática, repositorios con contenidos educativos digitales y planes de formación permanente para el profesorado, ofrecidos desde las administraciones educativas. Estas han producido una rápida “capacitación del profesorado en el uso y manejo de TIC para no perder el tren del progreso se recuerda constantemente, y ha servido a las autoridades educativas para forzar planes de formación que no han llegado a calar en la actividad profesional de los docentes” (Gutiérrez Martín, 2007, 150), pero no han respondido a las necesidades de una formación que capaciten para transformar la práctica educativa con ayuda de las TIC, ya que se han centrado en el dominio de aplicaciones informáticas (Paredes Labra, 2010, 55; Sancho et al., 2008, 16; Sánchez-Antolín, Ramos, y Sánchez Santamaría, 2014, 105). A su vez, para la formación inicial del profesorado de infantil y primaria, de carácter obligatorio, sólo existían unas recomendaciones genéricas que cada Universidad implementaba de forma distinta (organización y contenidos) (de Pablos Pons, 2013). Formación que "no fue pensada para una apuesta integradora, 'el estudio crítico y emancipador de las TIC', (...); fue 'formación piramidal, asistencial, no acreditativa' (...)” (Paredes Labra, 2013, 66) y que dejó de ser obligatoria en el curso 2009-10.

\subsection{La fugacidad del modelo 1:1 en España}

En 2009 se produjo un cambio en las políticas TIC con la introducción del modelo 1:1, a través del Programa Escuela 2.0, promovido por el Gobierno de España y destinado a todas las Comunidades Autónomas, formaba parte del Plan Español para el estímulo de la Economía y el Empleo. Este cambio se produjo, sobre todo, debido a la reducción del coste y peso de los equipos informáticos y a la disponibilidad de conectividad inalámbrica (Valiente González, 2011, 116); y siguiendo otros modelos, que ya se habían puesto en marcha siguiendo el modelo propuesto por Negroponte de "One Laptop for Child” (Angeriz, Curbelo, Folgar, y Gómez, 2012; Prats, 2013), tanto iberoamericanos (Plan Ceibal en Uruguay o Conectar Igualdad en Argentina) como europeos (Aulas digitales en Grecia o e.escola en Portugal) (Instituto de Tecnologías Educativas, 2011), a pesar de que no existían suficientes evidencias que expliquen qué fenómenos y factores, no sólo asociados a la introducción masiva de las TIC en los centros y aulas, favorecen los procesos de innovación y mejora educativa que se pueden producir trabajando con ordenadores (Area Moreira, 2010).

En este programa (eliminado por el gobierno conservador en su plan de recortes en 2012), participaban 15 de las 17 comunidades autónomas. Supuso la llegada masiva de ordenadores personales a las aulas y nuevas exigencias para el profesorado, sobre todo, de una competencia digital menos instrumental, "dentro de un modelo nuevo, que

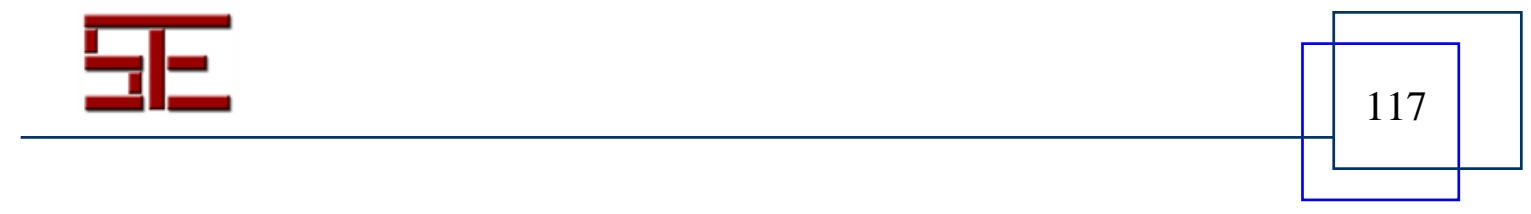




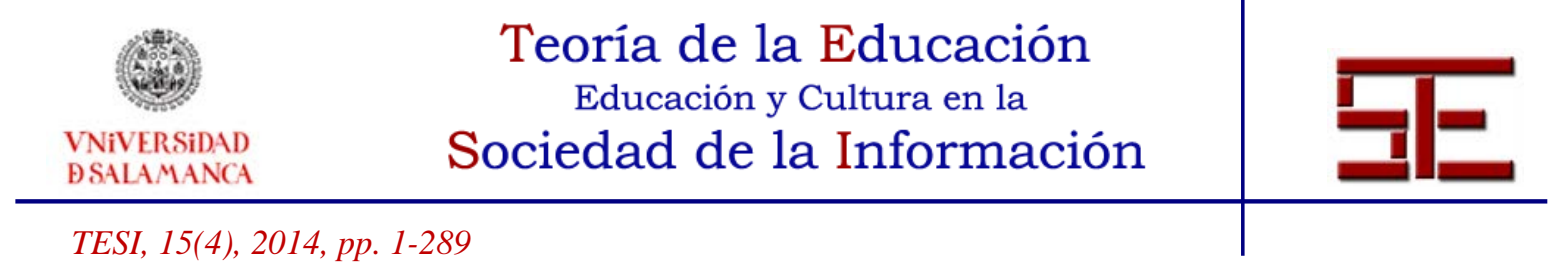

resquebraja otro más tradicional” (Sánchez-Antolín, Muñoz Álvarez, y Paredes Labra, $2013,196)$ y en el que las metodologías deben dejar de basarse en los libros de texto, las clases magistrales y los apuntes (Area Moreira, 2006, 224).

\subsection{Nuevas demandas de las políticas TIC}

Desde la eliminación del programa Escuela 2.0 en abril de 2012, las únicas actuaciones en materia educativa en relación con las TIC, se encontraban en la Agenda Digital para España, concretamente en una línea que trata de "desarrollar la economía digital para el crecimiento, la competitividad y la internacionalización de la empresa española” y más concretamente dentro del objetivo de "fortalecer la industria TIC mediante el desarrollo de proyectos tecnológicos en servicios públicos”. Ubicar las actuaciones de educación en esta línea y objetivo, y cuando el único indicador que se marca es "dotar de acceso de banda ancha ultrarrápida al 50\% de los centros educativos en 2015” (Gobierno de España, 2013a, 58), nos indica que más que tratarse de actuaciones pedagógicas, lo que se busca es un modelo de digitalización de la escuela económicamente sostenible y que fortalezca la industria TIC y la economía digital.

A finales de 2013, el Instituto Nacional de Tecnologías Educativas y de Formación del Profesorado (INTEF), presentó dos nuevos planes en relación con las TIC en las escuelas, el Plan de Cultura Digital en la Escuela y el Marco Estratégico de Desarrollo Profesional Docente (Instituto Nacional de Tecnologías Educativas y de Formación del Profesorado - INTEF, 2013c). Ambos planes, que se desarrollan en colaboración con las Comunidades Autónomas, y tiene muchos puntos en común con la iniciativa europea Apertura de la Educación, hacen referencia a la conectividad de los centros escolares, la interoperabilidad y estándares, la creación del espacio Procomún de contenidos en abierto, la competencia digital docente y el catálogo general de recursos educativos de pago Punto Neutro.

La creación de los dos portales de contenidos educativos digitales, uno de pago y otro gratuito, tal y como anunciaba Manuel Area en su conferencia "Políticas educativas TIC en España ¿de dónde venimos y a dónde vamos?” (2013), es uno de los fenómenos que se iba a producir en la política educativa española, la generación de un nuevo espacio en el que los materiales tradicionales (libros de texto) se irían sustituyendo por materiales digitales y en el que influyen no sólo aspectos pedagógicos, sino también derivados de los intereses comerciales de la industria editorial. El Punto Neutro (Instituto Nacional de Tecnologías Educativas y de Formación del Profesorado - INTEF, 2013b), contendrá el catálogo de recursos educativos de pago al que, entre otras características, se le atribuye el apoyo a la transformación de la educación y el cambio metodológico. Y EducaLAB (Instituto Nacional de Tecnologías Educativas y Formación del Profesorado - INTEF, 2013), que además de recursos educativos gratuitos online, a través de la plataforma

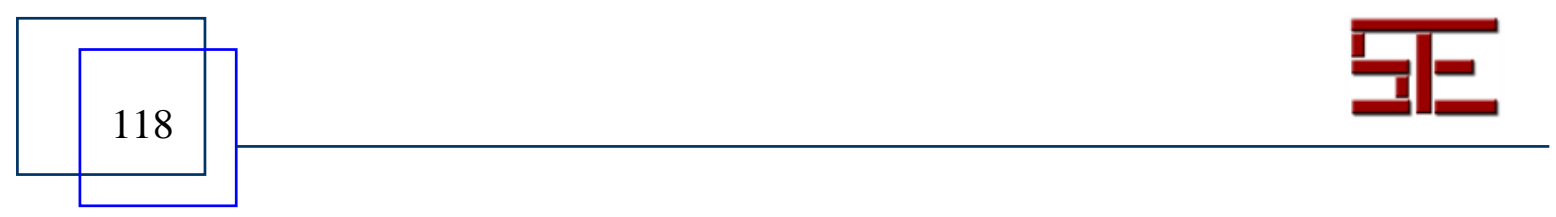




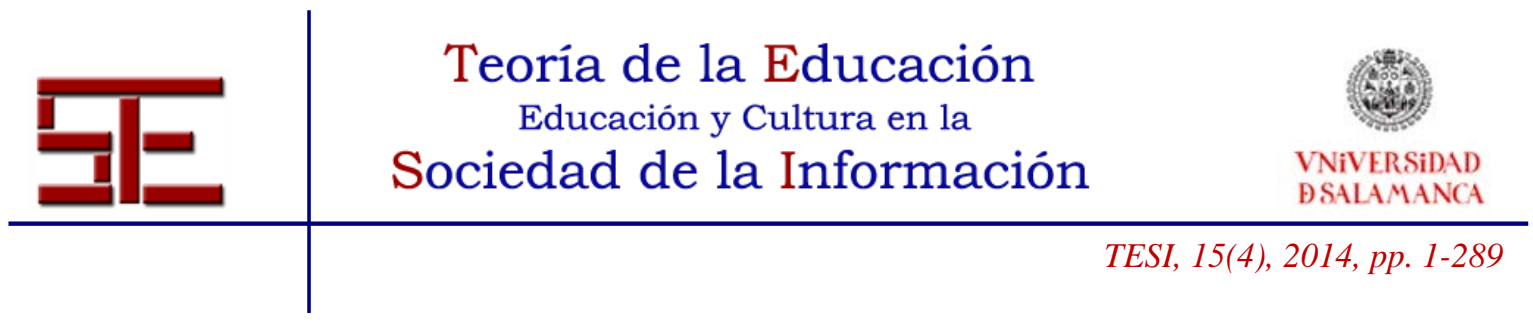

Procomún (Gobierno de España, n.d.), favorecerá la construcción de comunidades virtuales y la detección y difusión de prácticas innovadoras. Tal vez estemos ante la intromisión del sector empresarial en la enseñanza pública, con la excusa de los malos resultados que se obtienen y que por tanto hay que intervenir en ella (San Martín Alonso, 2009), o tal vez esté respondiendo más a intereses comerciales que a otros asociados a la integración de los medios como recursos en las aulas (Gutiérrez Martín y Tyner, 2012, 33).

En cualquier caso, las políticas educativas nacionales e internacionales apuntan a nuevas demandas para el profesorado que, entre otras muchas, pasan por una mayor competencia digital que les permita dar respuesta a la cultura digital en el aula a la que se apunta desde las estrategias educativas europeas, en las que se recomienda a los centros de educación "que adapten sus modelos de funcionamiento a la aparición de los recursos educativos abiertos (REA)” (Gobierno de España, 2013b) y en la LOMCE, en la que se reconoce el papel fundamental de las TIC en la formación del profesorado y la necesidad de crear "un marco común de referencia de competencia digital docente que oriente la formación permanente del profesorado y facilite el desarrollo de una cultura digital en el aula” (Gobierno de España, 2013a, 97899).

\section{POLÍTICAS TIC DE LA COMUNIDAD DE MADRID}

\subsection{La concreción del modelo 1:1 de la Comunidad de Madrid}

A partir de la generalización de la transferencia de competencias educativas a las Comunidades Autónomas, en la década de los 90, éstas han incluido entre sus políticas planes específicos para la integración curricular de las TIC en los centros educativos y para la formación de los docentes (Martín Hernández, 2011), como complemento a los proyectos Atenea y Mercurio, iniciados a mediados de los 80 (Area Moreira et al., 2012, 80). Estos planes han incluido medidas para el desarrollo de infraestructuras tecnológicas, portales educativos, repositorios de recursos, intranet para los centros y planes de formación para los docentes y gestores de los centros educativos (de Pablos Pons, Colás Bravo, y González Ramírez, 2010).

En la Comunidad de Madrid, desde los años 90, se vienen realizado acciones de alfabetización digital desde el ámbito de la educación no formal y de adultos (Paredes Labra, 2003). La región tiene competencias en educación e integración de las TIC desde 1999, dispone de una unidad del antiguo PNTIC muy activa.

Gracias al programa nacional, durante la década de los 90 la región recibió dotación tecnológica, tuvo un observatorio de recursos y tendencias, sus profesores recibieron formación permanente compartida, es verdad, con la labor de la UNED, hubo material

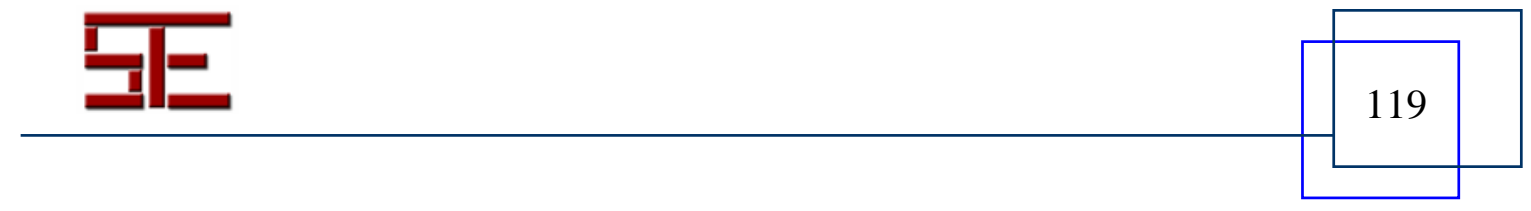




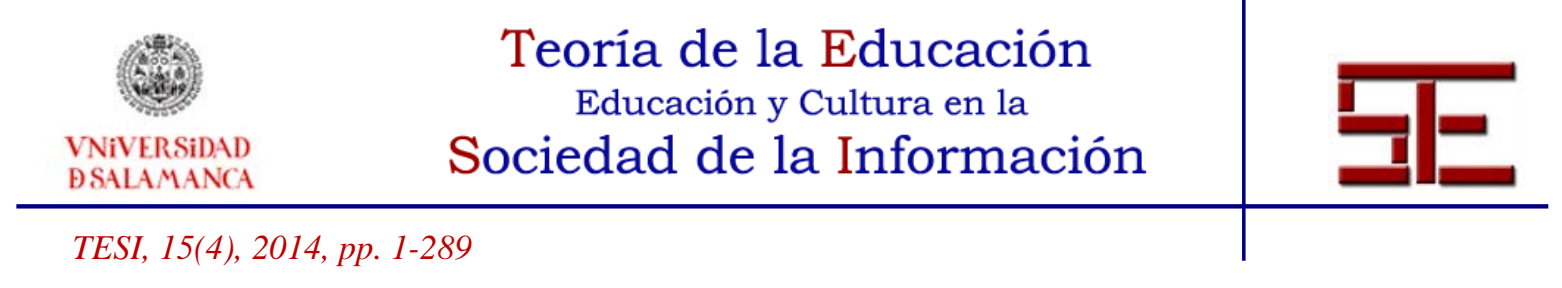

de paso, premios, repositorio, educación a distancia y un plan que se reprodujo a nivel regional cuando se obtuvieron las competencias para la gestión de la educación en este territorio.

No fue hasta abril de 2002 cuando se presentó el Plan Global para el desarrollo de las Tecnologías de la Información y la Comunicación en los centros docentes. En 2005 se creó el I Plan para el desarrollo de la Sociedad Digital y del Conocimiento en la Comunidad de Madrid.

En 2013, la Consejería de Educación, Juventud y Deporte publicó un resumen de las actuaciones que, desde las distintas unidades administrativas de la Comunidad de Madrid, se han realizado para el fomento del uso de las TIC en los centros docentes. Algunas de ellas hacen referencia a programas que se realizan en colaboración con el Ministerio de Educación (Agrega2 y Redined), la Comunidad Europea (eTwinning) o están dirigidas a la Enseñanza Superior (EMES). Aquí nos centraremos en las que hacen referencia a los centros de educación secundaria.

En el caso de la Comunidad de Madrid, la concreción del modelo 1:1 de la política nacional se denomina Institutos de Innovación Tecnológica (Comunidad de Madrid, 2010a) que se caracteriza por el tipo de dotación (terminales fijos en vez de portátiles) y por el alcance de la innovación (menos del $5 \%$ de los centros que imparten $1^{\circ}$ y $2^{\circ}$ de la E.S.O) (Paredes Labra, 2012a), en total, 15 Institutos.

La renuncia al proyecto del Ministerio de Educación, y a los 11 millones de euros de financiación, supuso detraer 15 millones de los 34,6 millones de euros, con los que estaba dotado el plan de mejora y modernización de tecnologías de la información y la comunicación en centros de educación secundaria (MIES), para modernizar los 15 institutos (Alcaide y Álvarez, 2010). Entre los motivos esgrimidos por la Consejera de Educación, Lucía Figar, para no acogerse al programa Escuela 2.0 se encontraban la falta de un plan de seguimiento y evaluación de los resultados que, en el caso de la Comunidad, será supervisado por la Consejería de Educación y en el que se incluyeron pruebas de evaluación específicas, y la imposibilidad de alfabetizar digitalmente en otros sistemas operativos que no fuesen Windows.

Entre los acuerdos firmados por la Consejería de Educación para el desarrollo de los Institutos de Innovación Tecnológica, se encontraba uno con Microsoft, por el cual la empresa ponía a disposición de las nuevas aulas las últimas versiones de software de Microsoft, formación continua al profesorado, contenidos digitales, herramientas para la creación de los mismos y la posibilidad de desarrollar programas de alfabetización digital para profesorado y alumnado (Microsoft Ibérica, 2010).

Una vez resuelta la convocatoria de selección de centros (cuatro en Madrid capital, cuatro en la zona sur, tres en la este, tres en la oeste y uno en la zona norte) (Comunidad de Madrid, 2010b) y firmados los convenios de colaboración con empresas, se inició el

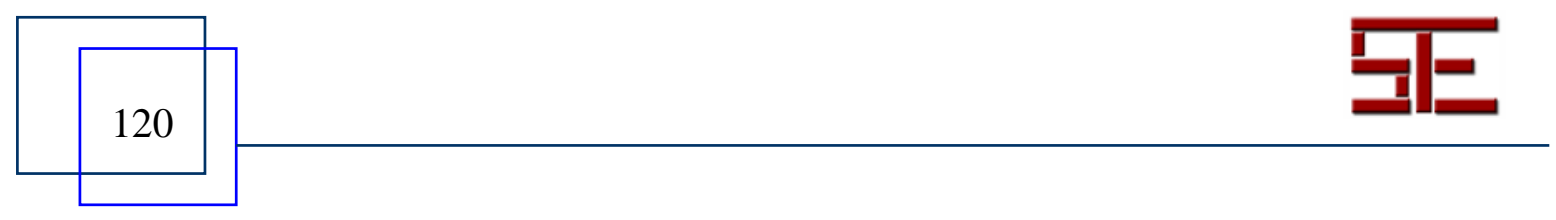




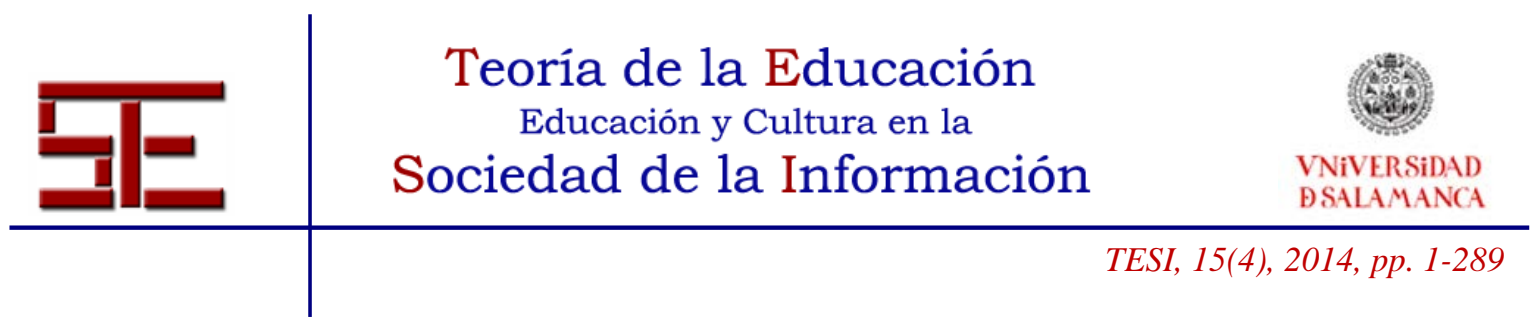

proyecto en el curso 2010-2011, con el objetivo de "contribuir a la mejora de los resultados académicos de los escolares madrileños, la Consejería de Educación se propone impulsar la utilización de las tecnologías de la información y de la comunicación como una herramienta de aprendizaje en la educación secundaria obligatoria” (Comunidad de Madrid, 2010a, 51).

Para la mejora de la calidad de la enseñanza, las políticas educativas de la Comunidad de Madrid han utilizado dos líneas de actuación fundamentales: de una parte, la promoción de la autonomía de los centros y de otra, la publicación de resultados de los centros educativos. En ambos casos se trata de fomentar la competitividad entre los centros y aumentar la libertad de elección de las familias (Prieto Egido y Villamor Manero, 2012). Esta iniciativa, propuesta y financiada desde la Comunidad de Madrid, se enmarca dentro de la primera línea de actuación, ofreciendo a los centros que participan la posibilidad de diferenciarse del resto, mediante una especialización curricular. Esta política tampoco prevé la participación de las familias, depende de cada centro la forma de implicar a las familias en el proyecto, que en algunos casos, se limita a la presentación del equipamiento, la metodología y la carga de trabajo que tendrá el alumnado, en las reuniones de principio de curso (Paredes Labra, 2012b).

Para la participación en esta iniciativa, además de la presentación del proyecto educativo de centro concretando la forma de implantación del programa para el curso 2010-2011, era necesario presentar entre otra documentación: certificación de las experiencias previas en la utilización de las TIC, relación de recursos tecnológicos del centro dedicados a la docencia, descripción de la formación previa del profesorado en el uso didáctico de las TIC, la aprobación del Consejo Escolar, el Claustro, los departamentos implicados en el proyecto y la propuesta del director del centro del profesor responsable del proyecto (Comunidad de Madrid, 2010a). En la valoración de las propuestas recibidas se tenía en cuenta por igual el grado de apoyo del Consejo Escolar, del Claustro y de los departamentos didácticos implicados (50\%) que el proyecto educativo presentado, la experiencia previa en la utilización de las TIC, los recursos tecnológicos disponibles y la formación previa del profesorado (50\%).

A la convocatoria se presentaron 208 de los 298 centros de educación secundaria de la Comunidad de Madrid (Microsoft Ibérica, 2010), de los que fueron seleccionados 15 (Comunidad de Madrid, 2010b). Estos centros han recibido la consideración de Institutos de Innovación Tecnológica, lo que les da derecho a contar con (Comunidad de Madrid, 2010a):

- Un profesor responsable del desarrollo del proyecto.

- Formación específica para la elaboración de materiales didácticos para el profesorado del proyecto.

- Aulas informáticas conectadas en red y con acceso a Internet (WIFI y banda

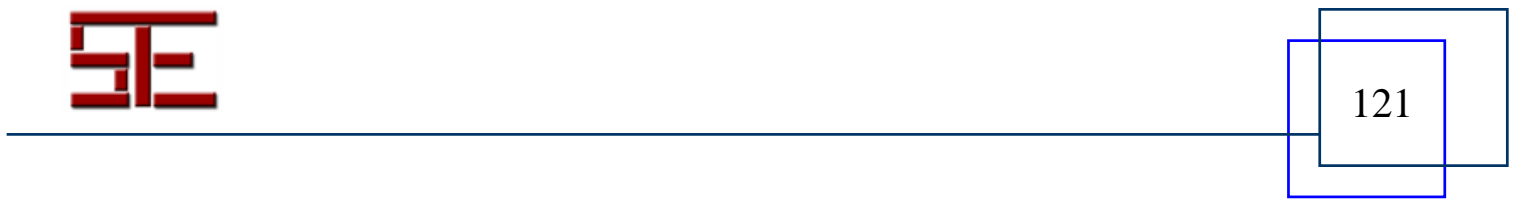




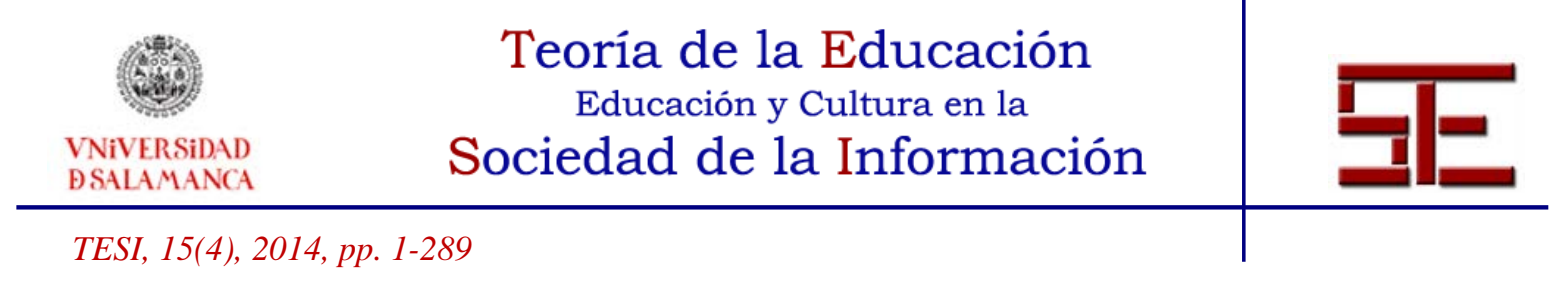

ancha) dotadas con un puesto informático por alumno (pupitres anclados al suelo y conectados a un servidor por cada seis puestos, normalmente en hileras que miran hacia la pizarra digital, preparadas para ocultar y desconectar la pantalla una vez bajada y con una bandeja extraíble para el ratón y el teclado), ordenador para el profesor, pizarras digitales y materiales didácticos específicos.

- Complementos retributivos para los responsables del proyecto y el profesorado implicado en el mismo. Estos complementos oscilan entre los 215,94€, para el coordinador del programa, y entre los 165,53€ y los 41,14€, en función de las horas semanales impartidas con TIC, para el resto del profesorado que participa en el programa (Comunidad de Madrid, 2010c).

Además de las ventajas que supone la consideración de Institutos de Innovación Tecnológica, la participación en el proyecto implicaba sumarse a los procesos de evaluación y seguimiento y la impartición, utilizando este nuevo entorno, de las asignaturas de Tecnología y Matemáticas y de al menos otras dos de entre, Ciencias de la naturaleza, Ciencias sociales, Geografía e Historia, Educación plástica y visual, Lengua extranjera y Lengua castellana y literatura (Quirino Vargas, 2011), hasta un máximo de ocho materias o asignaturas. En la distribución de la carga horaria de la asignatura de matemáticas y de las dos materias seleccionadas, al menos un 30\% debía realizarse utilizando las TIC como herramienta didáctica e incluir un plan detallado de evaluación de los procesos y resultados (Comunidad de Madrid, 2010a).

Finalmente, la Consejería de Educación de la Comunidad de Madrid (2010), en el plan estratégico de los Institutos de Innovación Tecnológica, destaca que la implantación de la enseñanza digital en la educación secundaria supondrá:

- Una mayor motivación para el aprendizaje y enseñanza individualizada.

- Una mayor atención a la diversidad y al alumnado con necesidades educativas especiales.

- Un mayor seguimiento y evaluación del proceso de aprendizaje.

- La potenciación de la competencia digital como herramienta para adquirir las competencias instrumentales básicas (comunicación lingüística y matemática).

\subsection{La vigencia del modelo 1:1 en Madrid}

A pesar de que, en el curso 2013/14, se han ampliado algunas aulas digitales en los institutos participantes, no se ha producido una ampliación del programa, sino que se ha puesto en marcha un nuevo proyecto piloto, para la incorporación de tabletas digitales Android y contenidos digitales, en dos Institutos, el IES Santamarca y el IES Arturo Soria, enmarcado dentro del Proyecto IDEA de la Fundación Albéniz, quien hace la

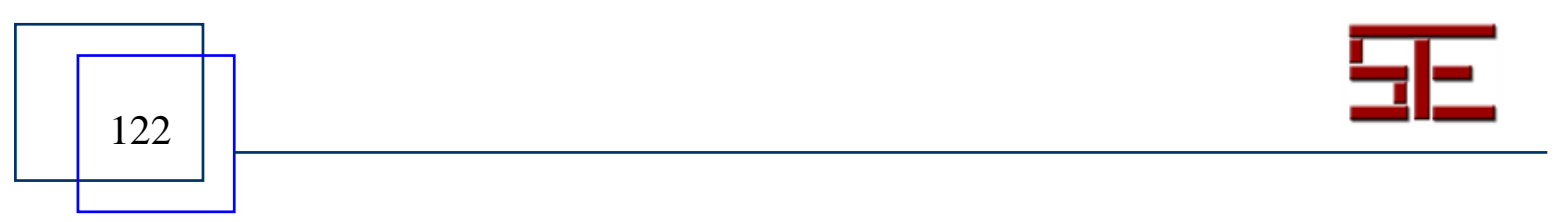




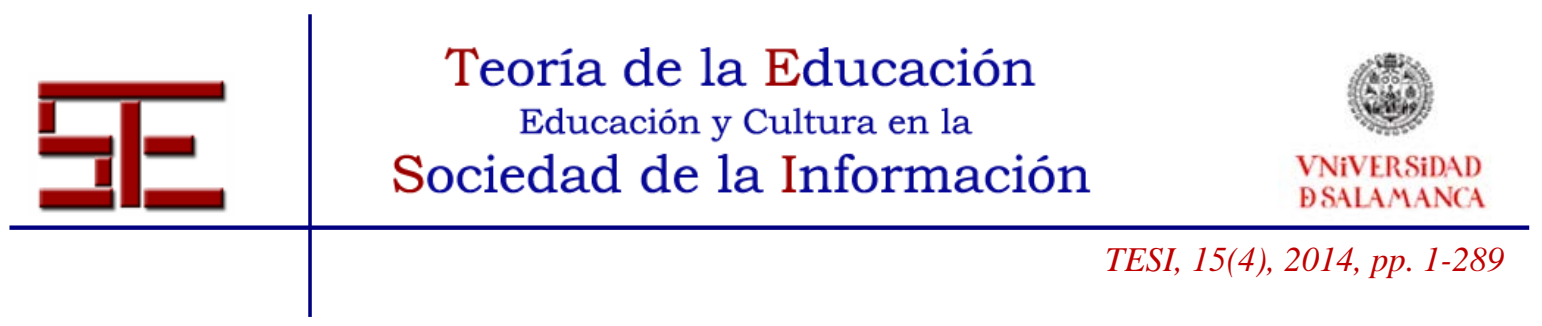

inversión, en colaboración con la Consejería de Educación, Juventud y Deporte (Rivas, 2012).

Para que el alumnado pueda participar en este proyecto, la familia se tiene que comprometer a la compra del dispositivo y las licencias de los libros digitales, a la reposición de la tableta si fuera necesario y a participar activamente en la gestión de las comunicaciones con el centro (IES Santamarca, 2013). En la hoja informativa del IES Santamarca, se aclara que la participación en el proyecto es voluntaria, sólo si el número de alumnos que no desean participar forman un grupo completo (30-33), en caso contrario, no podrán seguir con la opción de libros de texto.

\section{DISCUSIÓN}

El análisis comparado y la valoración de los tres niveles explicitados presentan, en términos generales, unos propósitos, unos medios y unos resultados, que se quieren aterrizar en este estudio en la realidad madrileña.

En el ámbito europeo, y en relación con los propósitos, conviene indicar que las temáticas europeas han sido unas políticas compasivas y solidarias preocupadas por los excluidos y colectivos en dificultades (Mangen, 2009), como la tercera edad, los discapacitados y los jóvenes, constantes en las convocatorias de ayudas de estudio y programas impulsados por Europa. Al mismo tiempo, se han perseguido ideales europeos como el mercado y la movilidad. Posiblemente no ha habido ideas sustantivas para el ámbito educativo.

En el ámbito Español, las temáticas abordadas por las políticas de los últimos 10 años se han armonizado con las europeas, buscando una financiación. Sin embargo, han sido las políticas educativas generales las que han acabado imponiendo su lógica en el papel de las TIC como recursos. Han estado demasiado centradas en lo escolar.

Se había previsto, en un principio, la alfabetización de los docentes como elemento clave de la puesta en marcha de estas innovaciones. La crisis económica, una evaluación apresurada de los efectos de la formación continua y cierta ingenuidad (como se ha puesto de manifiesto en el diseño de la formación inicial más reciente) hicieron pensar que no había que seguir realizando esta tarea alfabetizadora. Cierta retórica en los planes que se diseñan en la actualidad ofrece la formación continua a distancia como nueva solución.

Con respecto a los resultados, las innovaciones que han tenido lugar muestran un grado de ejecución y puesta en marcha muy similares. Las políticas nacionales han establecido observatorios, pero han estado más preocupados por las tendencias del mercado del software y dispositivos que por el estudio y contextualización de las medidas adoptadas en las escuelas. Los propósitos planteados en este tiempo y los medios empleados son

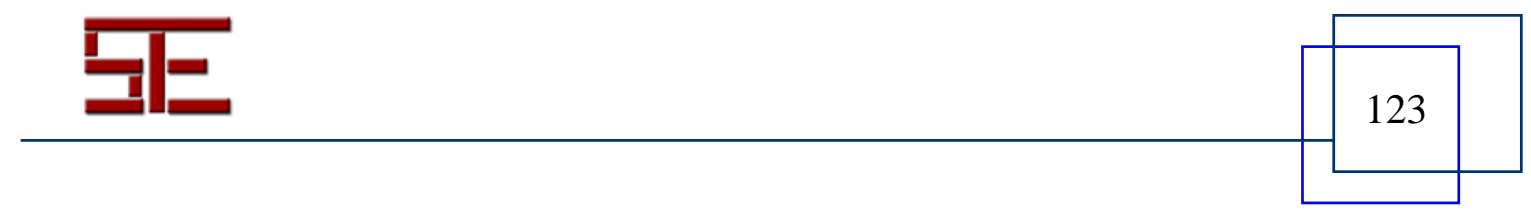




\section{\begin{tabular}{ccc}
$\begin{array}{c}\text { Teoría de la Educación } \\
\text { Educación y Cultura en la } \\
\text { VNiVERSIDAD } \\
\text { BSALAMANCA }\end{array}$ & Sociedad de la Información \\
\hline TESI, 15(4), 2014, pp. 1-289 &
\end{tabular}}

incongruentes. Cada política nacional ha estado denunciando la ingenuidad de la anterior, el desapego por el análisis de la realidad en la que se ponía en marcha. La sucesión de políticas evidencia una falta de evaluaciones de las políticas públicas en la introducción de las TIC en el sistema educativo.

En relación con Madrid, la falta de imaginación al respecto de los propósitos de la integración de las TIC en educación trajo una política dotacional que ahora se transforma en un instrumento para mejorar el rendimiento académico.

Se definió un sistema (dotación tecnológica, observatorio, formación, material de paso, premios, repositorio, educación a distancia) como parte de un plan que, descentralizado ya del todo con el comienzo de siglo, se reprodujo a nivel regional, y durante estos años se generaron dotaciones para el funcionamiento del mismo.

Se observan unos resultados discretos en cuanto a la vigencia y pujanza de una enseñanza transformada por las TIC en la región de Madrid.

Se integraron las TIC con cierto éxito pero cuando llegó una posibilidad de generalización (el modelo 1:1) no hubo ni voluntad política ni recursos más que para unas propuestas testimoniales, de escaparate.

Es particularmente preocupante que a estas alturas los medios que se han puesto en juego sean los productores de contenidos multimedia para las escuelas. Se ha dado por realizada toda la dotación, el resto de aspectos de las TIC en las escuelas se ha considerado resuelto por el mercado.

Otra cuestión preocupante es la formación del profesorado, pues se les ha requerido ya formados en TIC para poder apoyarlos a emprender una innovación y la adicional que se ofrece se vincula a una conocida empresa de software, introduciendo una lógica empresarial. Posiblemente haya que seguir apostando por la formación inicial y continua del profesorado, con más investigación sobre cómo funcionan estas innovaciones y nuevas soluciones que impliquen a los docentes.

Los programas de contenido social son externos, hay una obsesión por programas experimentales basados en dispositivos para la saturación tecnológica, sin modelo pedagógico y con objetivos coherentes con los imprimidos al sistema (resultados).

Las políticas pasaron de promover la alfabetización, la inclusión y la conectividad en el ámbito europeo a apostar por la adquisición de habilidades tecnológicas, primero, y se concretaron en la mejora del rendimiento en Madrid. Esta evolución acaba con la ilusión de la construcción de una educación humanista y deriva en un enfoque técnico plano. Esta cuestión se conecta con la perspectiva del currículo que empujan estas políticas, en un primer tiempo transversales y recientemente, al menos en Madrid, disciplinares.

Sorprende que durante más de 30 años no haya habido una vocación evaluadora e investigativa sobre los resultados de estas políticas. Al punto que la región de Madrid,

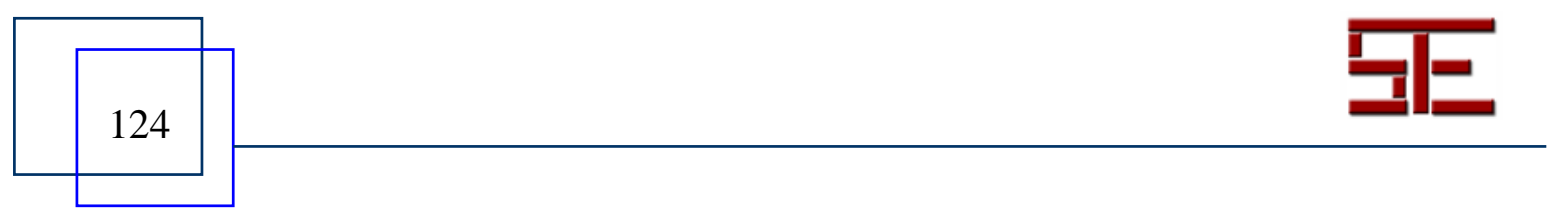




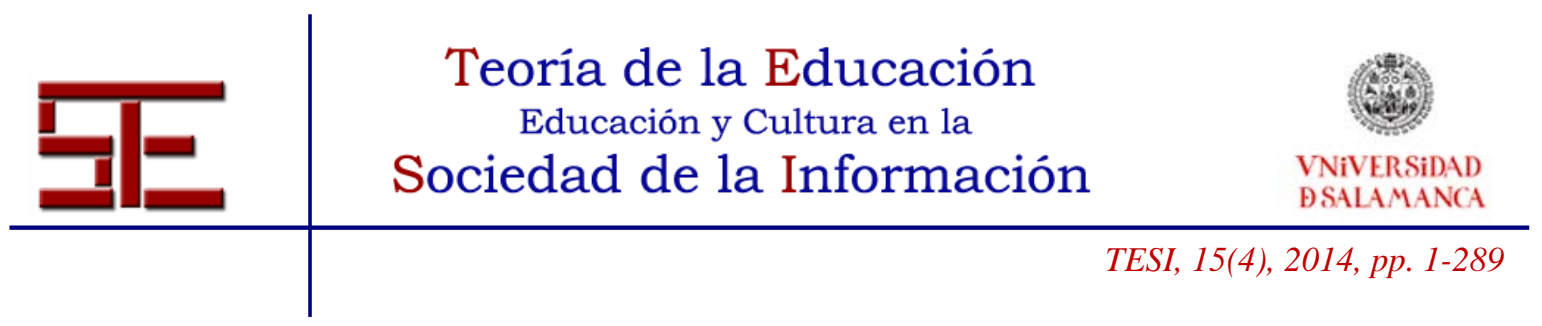

tras 30 años, decida poner en marcha políticas basadas en la eficacia de los dispositivos, algo que la investigación científica ya había demostrado poco relacionado con los resultados de la enseñanza en los inicios de la informática en la escuela.

Posiblemente han faltado ideas sustantivas para el cambio educativo. Hubieran convenido propósitos que hicieran dialogar lo escolar con otros horizontes para la ciudadanía, y de esta forma haber generado auténticos retos educativos que hubieran sido abordados con TIC en las escuelas. Progreso económico, competitividad, mercado, destrezas (trabajo en equipo), participación democrática, reforma de la administración, accesibilidad discapacidad, inclusión, juventud, personas mayores, cohesión social, alfabetización digital y conectividad se entrecruzan con calidad, sostenibilidad y resultados.

Los altos propósitos de las políticas, la insuficiencia de los medios empleados y la inadecuación de las medidas adoptadas para resolver los problemas planteados, el carácter estrella de unas innovaciones discretas, puede estar relacionada con la normalización de la existencia de algunos recursos, el agotamiento de las ideas sobre las posibilidades de la innovación y el decaimiento de unas políticas.

Se puede hablar de cierta improvisación de las políticas (particularmente en las acciones derivadas en Madrid). Las políticas del modelo 1:1 son, quizá, un salto, aunque discreto y efímero.

\section{REFERENCIAS}

Alcaide, S., y Álvarez, P. (2010, May 28). Aguirre mete en las aulas ordenadores más grandes que los de Zapatero. El País, p. 3. Madrid. Recuperado de http://elpais.com/diario/2010/05/28/madrid/1275045857_850215.html

Angeriz, E., Curbelo, D., Folgar, L., y Gómez, G. (2012). Plan CEIBAL en Uruguay. Una mirada universitaria sobre el impacto social y educativo. Campus Virtuales, 01(1), 65-78.

Area Moreira, M. (2006). Veinte años de políticas institucionales para incorporar las tecnologías de la información y comunicación al sistema escolar. En J. M. Sancho Gil (Ed.), Tecnologías para transformar la educación (pp. 199-232). Madrid: Akal.

Area Moreira, M. (2010). El proceso de integración y uso pedagógico de las TIC en los centros educativos. Un estudio de casos. Revista de Educación, 352, 77-97.

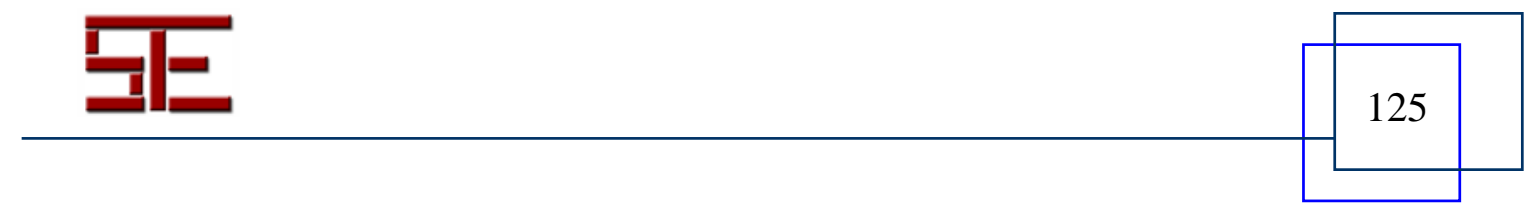




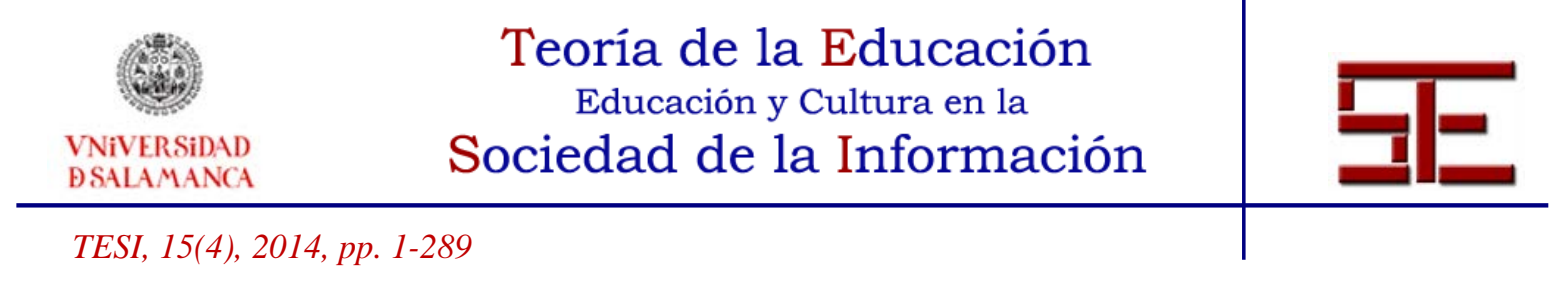

Area Moreira, M. (2012). Políticas educativas TIC en los sistemas escolares en Iberoamérica. Miradas desde las dos orillas. Campus Virtuales, 01(1), 7-9.

Area Moreira, M. (2013). Conferencia: “Políticas educativas TIC en España ¿de dónde venimos y a dónde vamos?” Recuperado 15 de Enero de 2014, de

http://ordenadoresenelaula.blogspot.com.es/2013/12/conferencia-politicas-educativastic-en.html

Area Moreira, M., Gutiérrez Martín, A., y Vidal Fernández, F. (2012). Alfabetización digital y competencias informacionales. Barcelona: Ariel.

Bangemann. (1994). Informe sobre Europa y la sociedad global de la información. Boletín de La Unión Europea. Suplemento, (2), 5-40.

Burbules, N. C., y Callister, T. A. (2001). Educación: riesgos y promesas de las nuevas tecnologías de la información. Buenos Aires: Ediciones Granica SA.

Cela, J. (2005). Sociedad del conocimiento y sociedad global de la información: Implantación y desarrollo en España. Documentación de Las Ciencias de La Información, 28, 147-158.

Cobo Romaní, C. (2010). ¿Y si las nuevas tecnologías no fueran la respuesta? En A. Piscitelli, I. Adaime, y I. Binder (Eds.), El proyecto Facebook y la posuniversidad. Sistemas operativos sociales y entornos abiertos de aprendizaje. Barcelona: Ariel.

Comisión de las Comunidades Europeas. (1995). Libro blanco sobre la educación y la formación: enseñar y aprender. Hacia la soceidad cognitiva. Oficina de Publicaciones Oficiales de las Comunidades Europeas. Recuperado 16 de Enero de 2013, de http://eurlex.europa.eu/LexUriServ/LexUriServ.do?uri=COM:1995:0590:FIN:ES:PDF

Comisión de las Comunidades Europeas. (2000). eEurope 2002. Una sociedad de la información para todos. Plan de acción preparado por el Consejo y la Comisión Europea para el Consejo Europeo de Feira 19-20 de junio de 2000. Recuperado 22 de Noviembre de 2012, de http://ec.europa.eu/information_society/eeurope/i2010/docs/2002/action_plan/actionpl an_es.pdf

Comisión de las Comunidades Europeas. (2002). Comunicación de la Comisión al Consejo, al Parlamento Europeo, al Comité Económico y Social y al Comité de las regiones. eEurope 2005: Una sociedad de la información para todos. Recuperado 15 de

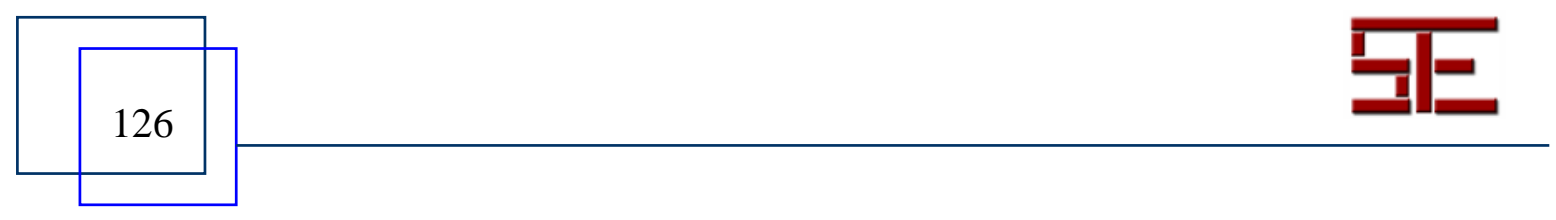




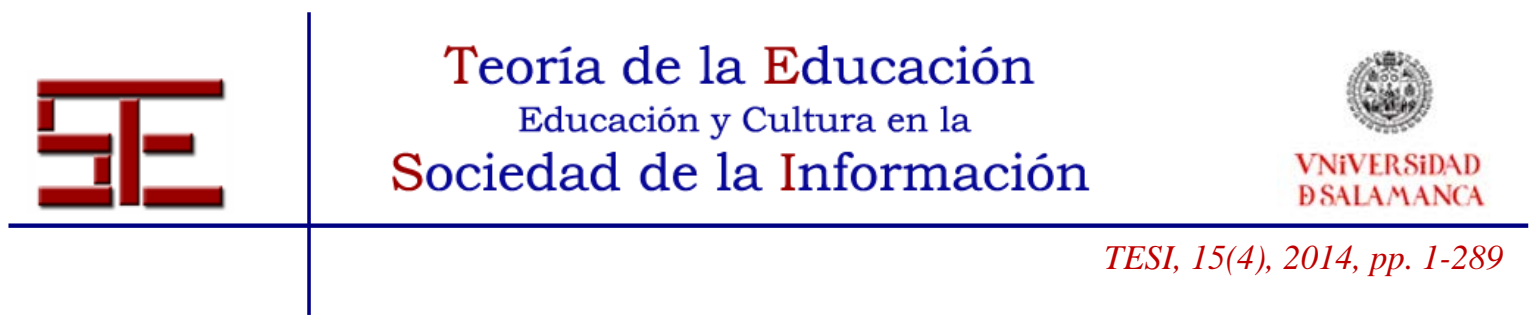

Noviembre de 2012, de http://eur-

lex.europa.eu/LexUriServ/LexUriServ.do?uri=CELEX:52002DC0263:ES:NOT

Comisión de las Comunidades Europeas. (2004a). Comunicación de la Comisión al Consejo, al Parlamento Europeo, al Comité Económico y Social Europeo y al Comité de las Regiones. Retos para la sociedad de la información europea con posterioridad a 2005. Recuperado 24 de Noviembre de 2012, de http://eurlex.europa.eu/LexUriServ/LexUriServ.do?uri=CELEX:52004DC0757:ES:HTML

Comisión de las Comunidades Europeas. (2004b). Revisión intermedia del Plan de acción eEurope 2005. Recuperado 18 de Marzo 2012, de http://eur-lex.europa.eu/legalcontent/ES/TXT/PDF/?uri=CELEX:52004DC0108\&from=ES

Comisión de las Comunidades Europeas. (2005). Comunicación de la Comisión al Consejo, al Parlamento Europeo y al Comité Ecocómico y social Europe y al Comité de las Regiones. i2010 - Una sociedad de la información europea para el crecimiento y el empleo. Recuperado 24 de Noviembre de 2012, de http://eur-

lex.europa.eu/LexUriServ/LexUriServ.do?uri=COM:2005:0229:FIN:ES:PDF

Comisión de las Comunidades Europeas. (2006). Comunicación de la Comisión al Consejo, al Parlamento Europeo, al Comité Económico y Social y al Comité de las Regiones. Iniciativa i2010 - Primer Informe Anual sobre la Sociedad de la Información Europea. Recuperado 05 de Diciembre de 2012, de http://eur-

lex.europa.eu/LexUriServ/LexUriServ.do?uri=COM:2006:0215:FIN:ES:PDF

Comisión de las Comunidades Europeas. (2007). Comunicación de la Comisión al Consejo, al Parlamento Europeo, al Comité Económico y Social y al Comité de las Regiones. Iniciativa Europea i2010 para la inclusión digital. «Participar en la sociedad de la información». Recuperado 22 de Noviembre de 2012, de

http://ec.europa.eu/information_society/activities/einclusion/docs/i2010_initiative/com m_native_com_2007_0694_f_es_acte.pdf

Comisión de las Comunidades Europeas. (2009). Evaluación final del plan de acción eEurope 2005 y del programa plurianual (2003-2006) para el seguimiento del plan de acción eEurope 2005, la difusión de las buenas prácticas y la mejora de la seguridad de las redes y la información (Modinis). Recuperado 09 de Julio de 2014, de http://eurlex.europa.eu/legal-content/ES/TXT/PDF/?uri=CELEX:52009DC0432\&from=ES

Comisión Europea. (2006). Ministerial Declaration. ICT Riga, 11-13 june 2006.

Recuperado 01 de Junio de 2013, de http://ec.europa.eu/information_society/events/ict_riga_2006/doc/declaration_riga.pdf

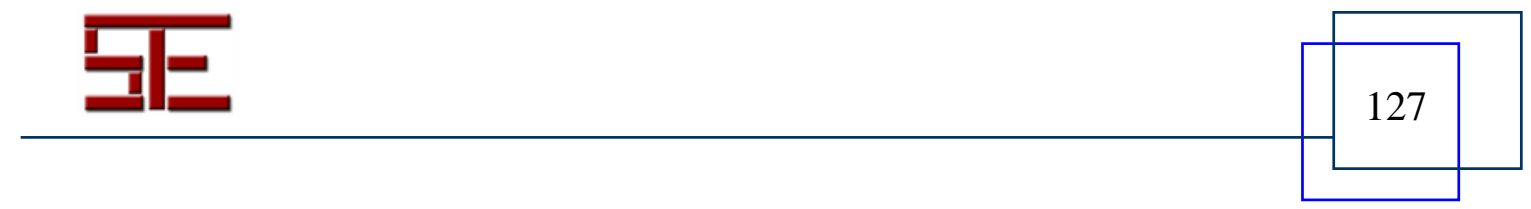




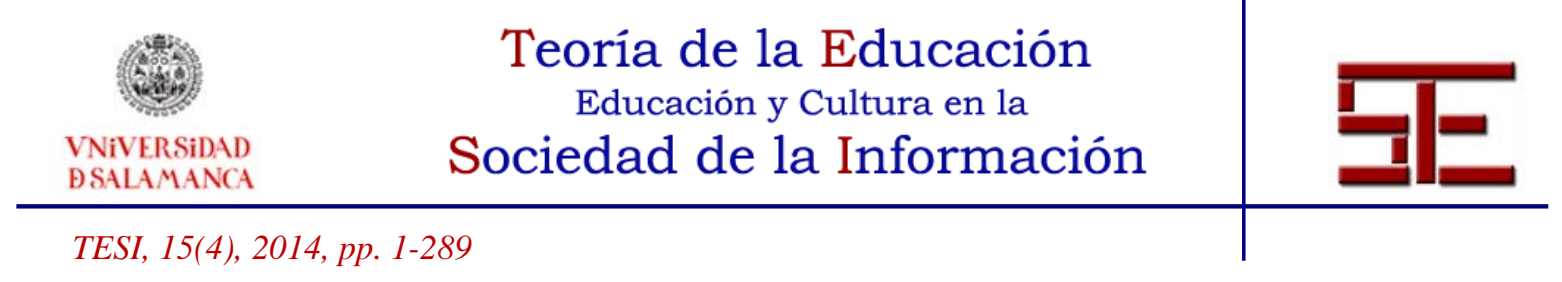

Comisión Europea. (2010a). Comunicación de la Comisión al Consejo, al Parlamento Europeo, al Comité Económico y Social y al Comité de las Regiones. Una Agenda Digital para Europa. Recuperado 25 de Noviembre de 2012, de http://eurlex.europa.eu/LexUriServ/LexUriServ.do?uri=COM:2010:0245:FIN:ES:PDF

Comisión Europea. (2010b). Europa 2020. Una Estrategia para un crecimiento inteligente, sostenible e integrador. Recuperado 05 de Diciembre de 2012, de http://ec.europa.eu/commission_20102014/president/news/documents/pdf/20100303_1_es.pdf

Comisión Europea. (2014). Erasmus+. Recuperado 08 de Febrero de 2014, de http://ec.europa.eu/programmes/erasmus-plus/index_es.htm

Comunidad de Madrid. (2010a). ORDEN 1275/2010, de 8 de marzo, por la que se implanta el proyecto de institutos de innovación tecnológica en la Comunidad de Madrid. Boletín Oficial de la Comunidad de Madrid. Recuperado 01 de Septiembre de 2013, de http://w3.bocm.es/boletin/CM_Orden_BOCM/2010/03/18/BOCM-2010031813,1.PDF

Comunidad de Madrid. (2010b). ORDEN 3369/2010, de 16 de junio, por la que se resuelve la convocatoria para la selección de Institutos de Educación Secundaria en los que se implantará el proyecto de institutos de innovación tecnológica en la Comunidad de Madrid. Boletín Oficial de la Comunidad de Madrid. Recuperado 01 de Septiembre de 2013, de http://w3.bocm.es/boletin/CM_Orden_BOCM/667/635/BOCM-2010072136.PDF

Comunidad de Madrid. (2010c). Orden de 1 de octubre de 2010, de la Consejería de Economía y Hacienda, por la que se establecen criterios objetivos para la asignación de productividad a los funcionarios de Cuerpos Docentes no Universitarios por la participación en programas que impliqu. Boletín Oficial de la Comunidad de Madrid. Recuperado 12 de Septiembre de 2013, de http://www.madrid.org/wleg/servlet/Servidor?opcion=VerHtml\&nmnorma=6812\&cdest ado $=\mathrm{P}$

Consejería de Educación de la Comunidad de Madrid. (2010). Institutos de innovación tecnológica en la Comunidad de Madrid. Recuperado 01 de Septiembre de 2013, de http://ies.rosachacel.colmenarviejo.educa.madrid.org/documentos/chatii/IIT.pdf

Consejo de Europa. (2000). Conclusiones de la Presidencia. Consejo Eurpeo de Lisboa 23 y 24 de marzo de 2000. Recuperado 09 de Julio de 2014, de http://www.minhap.gob.es/Documentacion/Publico/SGPEDC/Estrategia de Lisboa.pdf

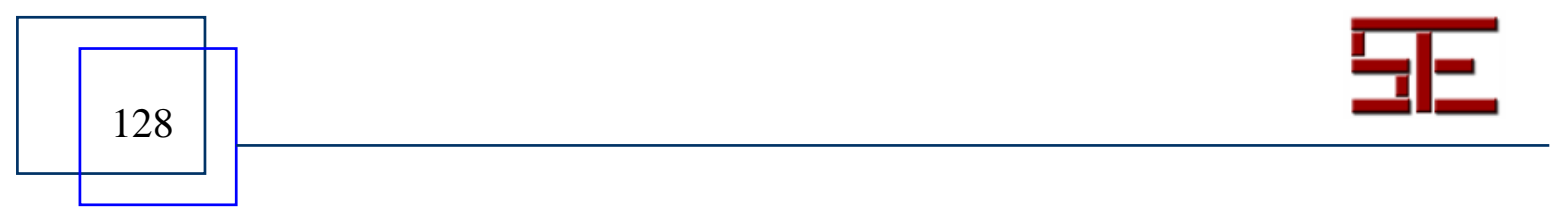




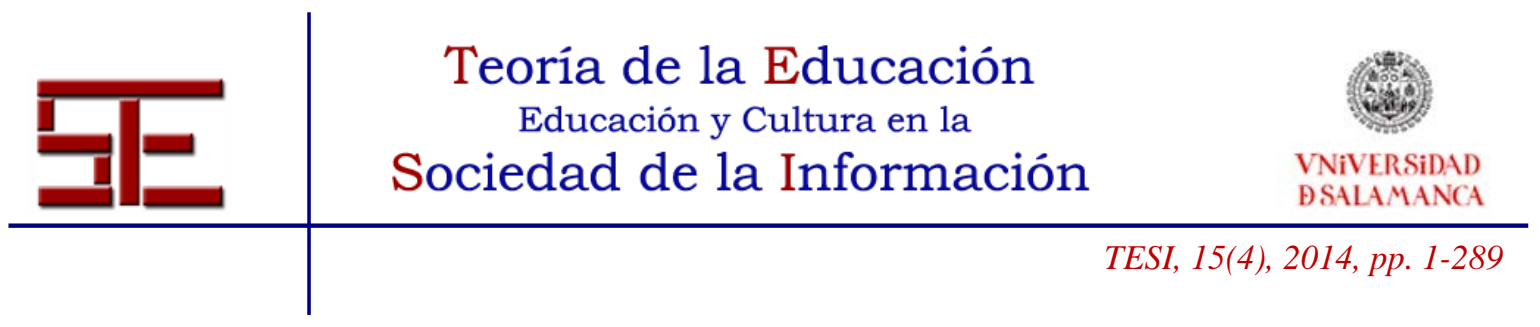

De Pablos Pons, J. (2013). La formación e investigación en el campo de la Tecnología Educativa. Revista Fuentes, 13, 9-16.

De Pablos Pons, J., Colás Bravo, P., y González Ramírez, T. (2010). Factores facilitadores de la innovación con TIC en los centros escolares. Un análisis comparativo entre diferentes políticas educativas autonómicas. Revista de Educación, 352(MayoAgosto), 23-51.

Del Blanco Diez, L. (1989). Proyecto Mercurio: Un instrumento institucional para impulsar la introducción de los medios audiovisuales en el currículo. Comunicación, Lenguaje Y Educación, 1, 95-101.

Diario Oficial de la Unión Europea. (2013). Reglamento (UE) No 1288/2013 del Parlamento Europeo y del Consejo de 11 de diciembre de 2013 por el que se crea el programa «Erasmus+», de educación, formación, juventud y deporte de la Unión y por el que se derogan las Decisiones n ${ }^{0}$ 1719/2006/CE, 1720/2. Recuperado 08 de Febrero de 2014, de

http://www.juventudenaccion.injuve.es/opencms/export/download/documentacion/Eras musPlusReglamento20_12_2013.pdf

Echeverría, J., y Unceta, A. (2012). Ciudadanía y participación en el espacio electrónico Europeo. ARBOR, 188(756), 725-732. doi:10.3989/arbor.2012.756n4007

Fernández Prieto, M. S. (2001). Las nuevas tecnologías en la educación: análisis de modelos de aplicación. Universidad Autónoma. Departamento de Didáctica y Teoría de la Educación.

Ferreiro Alonso, A. (2011). El desarrollo del concepto de competencia digital en el currículum de las enseñanzas obligatorias de Galicia. Innovación Educativa, 21, 151159.

Gobierno de España. (n.d.). Procomún. Recuperado 16 de Enero de 2014, de http://educalab.es/proyectos/procomun

Gobierno de España. (2005). Anexo II. Medidas por áreas de actuación 2007-2010. PlanAvanz@. Recuperado 24 de Noviembre de 2012, de https://www.planavanza.es/InformacionGeneral/PlanAvanza1/Descargas/2a392d4f65d9 404fb83fc3d2f64eceadplan_avanza_documento_completo.pdf

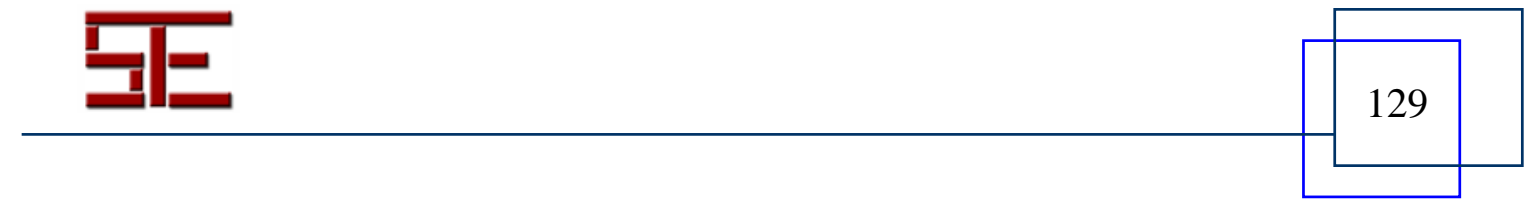




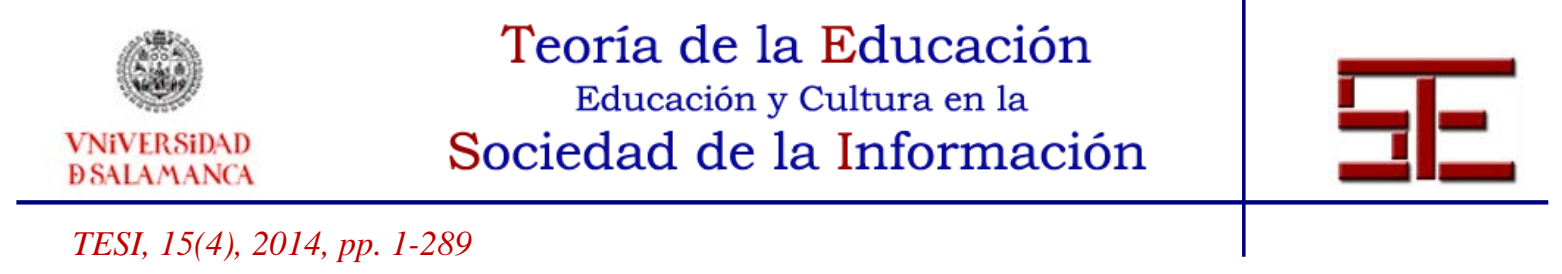

Gobierno de España. (2013a). Agenda Digital para España. Recuperado 20 de Septiembre de 2013, de http://www.agendadigital.gob.es/agendadigital/recursos/Recursos/1. Versión definitiva/Agenda_Digital_para_Espana.pdf

Gobierno de España. (2013b). Replantear la Educación: Nueva estrategia de la Comisión Europea. TIC. Recuperado 12 de Enero de 2014, de http://recursostic.educacion.es/blogs/europa/index.php/2012/11/30/replantear-laeducacion-nueva-estrategia-de-la-comision-europea-tic

Gutiérrez Martín, A. (2007). Integración curricular de las TIC y educación para los medios en la sociedad del conocimiento. Revista Iberoamericana de Educación, 45, 141-156.

Gutiérrez Martín, A., y Tyner, K. (2012). Educación para los medios, alfabetización mediática y competencia digital. Revista Comunicar, 19(38), 31-39.

doi:http://dx.doi.org/10.3916/C38-2012-02-03

IES Santamarca. (2013). El proyecto IDEA en el IES Santamarca. Recuperado 19 de Diciembre de 2013, de

http://www.educa.madrid.org/web/ies.santamarca.madrid/novedades/informacionfamili asproyectoiidea.pdf

Instituto de Tecnologías Educativas. (2011). Iniciativas 1:1 (pp. 1-30). Recuperado de http://recursostic.educacion.es/blogs/europa/media/blogs/europa/informes/Informe_1a1 _mundial_ITE_octubre_2011.pdf

Instituto Nacional de Tecnologías Educativas y de Formación del Profesorado - INTEF. (2013a). La Comisión Europea presenta “Apertura de la Educación.” Recuperado 15 de Enero de 2014, de http://blog.educalab.es/intef/2013/09/27/la-comision-europeapresenta-apertura-de-la-educacion/

Instituto Nacional de Tecnologías Educativas y de Formación del Profesorado - INTEF. (2013b). Punto Neutro: Catálogo de recursos educativos de pago. Recuperado 14 de Enero de 2014, de http://blog.educalab.es/intef/2013/10/11/punto-neutro-catalogo-derecursos-educativos-de-pago/

Instituto Nacional de Tecnologías Educativas y de Formación del Profesorado - INTEF. (2013c). Tecnologías y formación: proyectos del INTEF. Recuperado 15 de Enero de 2014, from http://blog.educalab.es/intef/2013/04/08/tecnologias-y-formacion-aplicadasa-la-educacion/

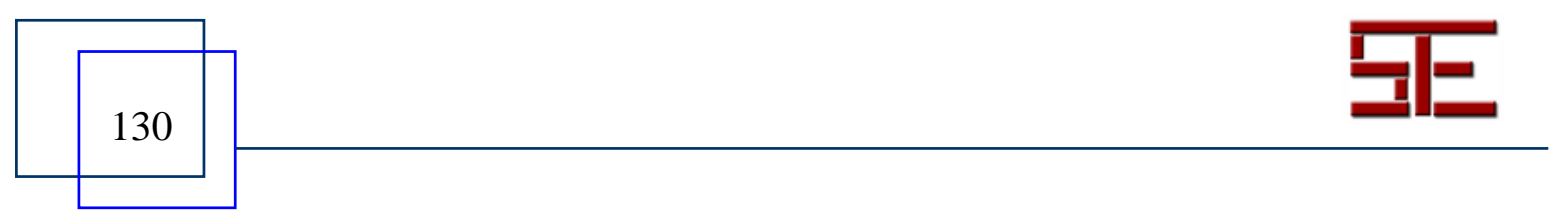




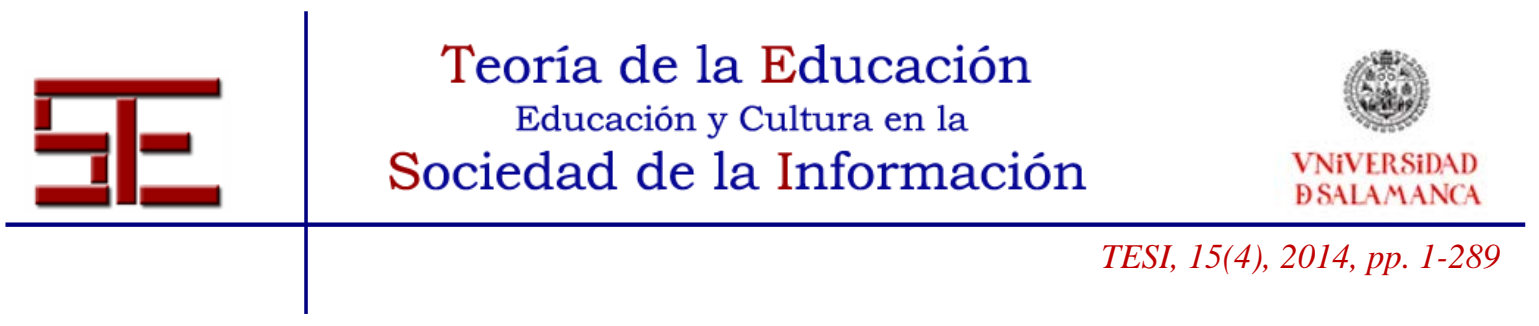

Instituto Nacional de Tecnologías Educativas y Formación del Profesorado - INTEF. (2013). Nuevo portal: EDUCALAB. Recuperado 12 de Enero de 2014, de http://www.ite.educacion.es/es/inicio/noticias-de-interes/11-nuevo-portal-educalab

Kok, W. (2004). Hacer frente al desafío: La estrategia de Lisboa para el crecimiento y el empleo. Bruselas: Comisión Europea.

Madrid Izquierdo, J. M. (2007). La política educativa de la Unión Europea al servicio del desarrollo económico con cohesión social. Revista Española de Educación Comparada, 13, 253-284.

Mangen, S. (2009). Europe and the Welfare State since 1945. In K. Larres (Ed.), A Companion to Europe Since 1945 (pp. 472-500). Chichester: Willey-Blackwell.

Martín Hernández, S. (2011). Escuela 2.0: Panorama Actual de la situación del programa. Recuperado 15 de Julio de 2013, de http://scopeo.usal.es/wpcontent/uploads/2012/10/Ponencia_escuela2.0.pdf

Microsoft Ibérica. (2010). La Comunidad lidera la introducción de las TIC en Secundaria con los Institutos de Innovación Tecnológica. Recuperado 13 de Septiembre de 2013, de http://www.microsoft.com/spain/prensa/noticia.aspx?infoid=/2010/11/n011Madrid-secundaria-TIC

OECD. (2010). Are the new millennium learners making the grade? Technology use and educational performance in PISA 2006. París. doi:10.1787/9789264076044-en

Paredes Labra, J. (2003). La formación en nuevas tecnologías en el ámbito de la educación no formal y de adultos. Experiencias en Madrid. Comunicación Y Pedagogía: Nuevas Tecnologías Y Recursos Didácticos, (186), 33-42.

Paredes Labra, J. (2010). Innovadores en espacios reinstrumentalizados. Aproximaciones etnográficas y narrativas a los centros innovadores con TIC en educación primaria y secundaria. Revista Iberoamericana Sobre Calidad, Eficacia $Y$ Cambio En Educación, 8(1), 46-62.

Paredes Labra, J. (2012a). La puesta en marcha del modelo 1a1 y la vida en las aulas. Análisis de 8 casos en la Comunidad de Madrid. En Jornadas Universitarias de Tecnología Educativa 2012 (pp. 1-8).

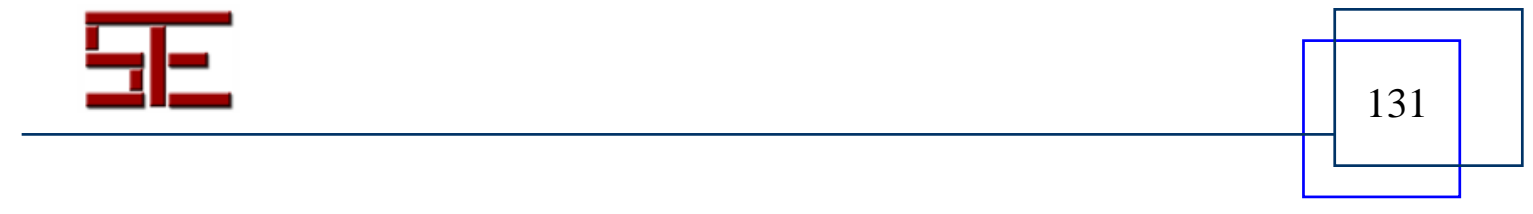




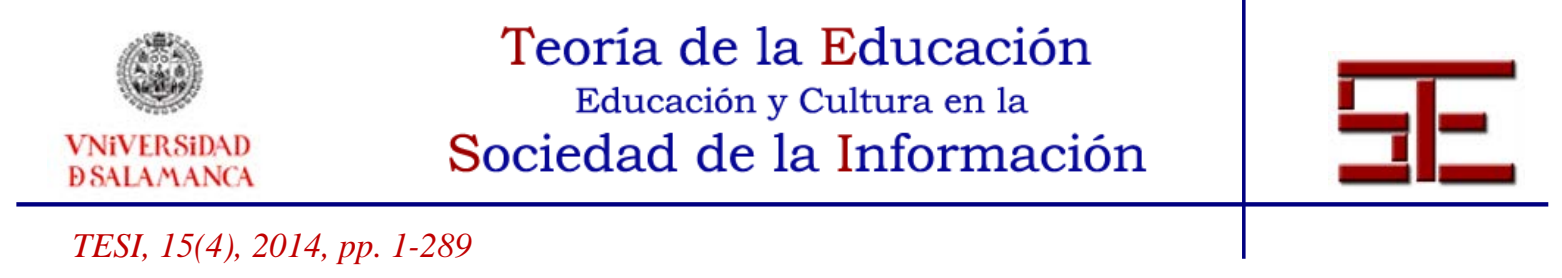

Paredes Labra, J. (2012b). Políticas educativas neoliberales para la integración de las TIC en educación. El caso de Madrid (España). Campus Virtuales, 01(1), 11-20.

Paredes Labra, J. (2013). Políticas educativas públicas sobre TIC en España. Tres décadas donde los docentes universitarios influyeron en el cambio educativo. Revista Fuentes, 13, 45-78.

Piscitelli, A. (2010). Edupunk, maestros ignorantes, educación invisible y el proyecto Facebook. En A. Piscitelli, I. Adaime, y I. Binder (Eds.), El proyecto Facebook y la posuniversidad. Sistemas operativos sociales y entornos abiertos de aprendizaje (pp. 320). Barcelona: Ariel.

Prats, E. (2013). Jugando a ser dioses: puntos críticos de las tecnologías en educación. Teoría de La Educación: Educación Y Cultura En La Sociedad de La Información, 14(3), 101-120. Recuperado de http://campus.usal.es/ revistas_trabajo/index.php/revistatesi/article/view/11353/11770

Prieto Egido, M., y Villamor Manero, P. (2012). Libertad de elección, competencia y calidad: las políticas educativas de la comunidad de Madrid. Profesorado. Revista de Currículum Y Formación Del Profesorado, 16(3), 127-144.

Quirino Vargas, J. (2011). Actuaciones en materia de tecnologías de la información y la comunicación desarrolladas por la Consejería de Educación de la Comunidad de Madrid. En Escuela 2.0 (II). Madrid. Recuperado de http://www.socinfo.es/contenido/seminarios/escuela20/comunidad_madrid.pdf

Rivas, T. G. (2012). Educación ensaya con tabletas en lugar de libros de texto en dos institutos públicos. ABC.es. Recuperado de http://www.abc.es/localmadrid/20121119/abci-educacion-tabletas-colegios-201211182107.html

San Martín Alonso, Á. (2009). La escuela enredada: formas de participación escolar en la sociedad de la información. Barcelona: Gedisa.

Sánchez-Antolín, P., Muñoz Álvarez, T., y Paredes Labra, J. (2013). Competencia digital de los estudiantes. Qué hacen los profesores de secundaria para alcanzarla en el modelo 1a1 de la Comunidad de Madrid. En M. Area Moreira (Ed.), Políticas educativas y buenas prácticas TIC. II Simposio internacional SITIC. Tenerife: Laboratorio de Educación y Nuevas Tecnologías de la Universidad de la Laguna (EDULLAB) (pp. 186-196). Recuperado de http://edullab.webs.ull.es/wordpress/wpcontent/uploads/2013/12/ACTAS-SITIC-

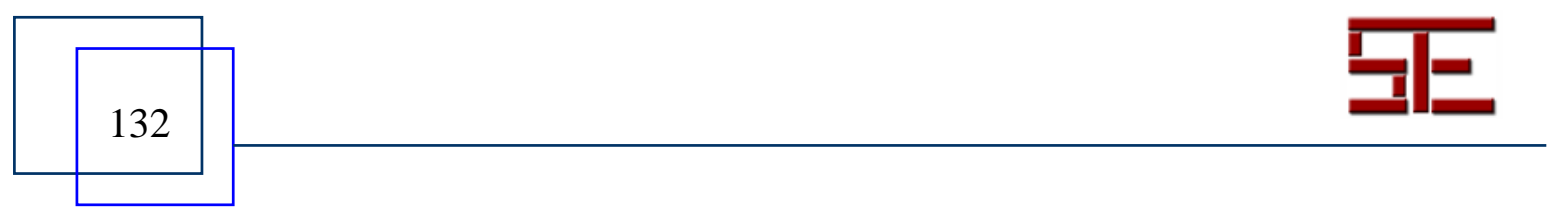




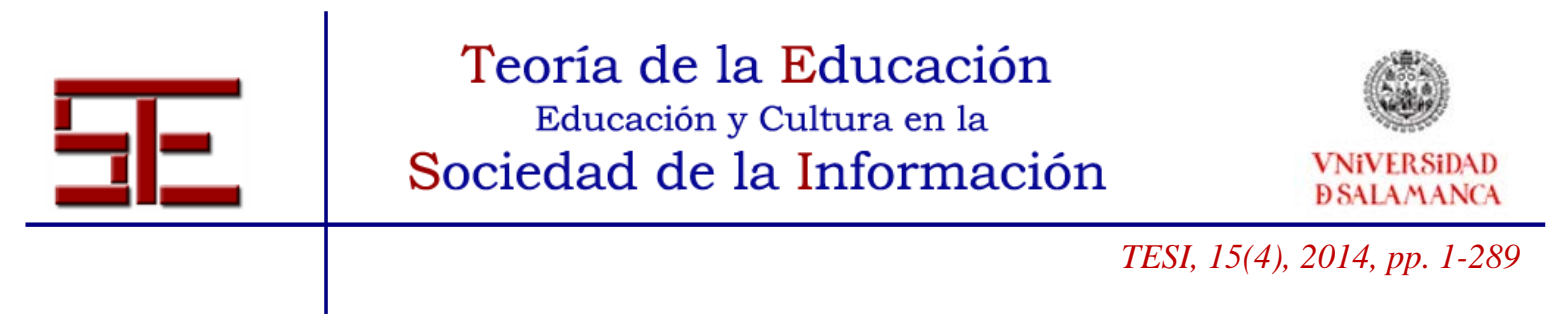

Sánchez-Antolín, P., Ramos, F. J., y Sánchez Santamaría, J. (2014). Formación continua y competencia digital docente: el caso de la Comunidad de Madrid. Revista Iberoamericana de Educación, 65, 91-110.

Sancho, J. M., Ornellas, A., Sánchez, J. A., Alonso, C., y Bosco, A. (2008). La formación del profesorado en el uso educativo de las TIC: una aproximación desde la política educativa. Praxis Educativa, 12, 10-22.

Segura, M., Candioti, C., y Medina, J. (2007). Las TIC en la Educación: panorama internacional y situación Española. Recuperado 20 de Enero de 2013, de http://www.fundacionsantillana.com/upload/ficheros/paginas/200906/xxii_semana_mon ografica.pdf

Somekh, B. (2000). New Technology and Learning: Policy and Practice in the UK, 1980-2010. Education and Information Technologies, 5(1), 19-37.

Soto Carballo, J. (2007). Políticas educativas y nuevos contextos de intervención en relación a las TIC. Panorama actual en el ámbito europeo y español. Revista de Investigación En Educación, 4, 4-21.

Valiente González, O. (2011). Los modelos 1:1 en Educación. Prácticas internacionales, evidencia comparada e implicaciones políticas. Revista Iberoamericana de Educación, 56, 113-134.

Valle López, J. M. (2004). La política educativa de la Unión Europea: fundamentos, evolución histórica y propuesta de un modelo para su análisis crítico. Revista Española de Educación Comparada, 10, 17-59.

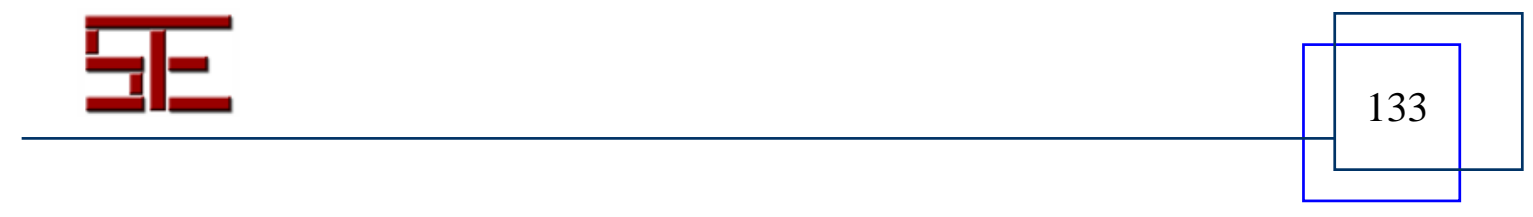

